\title{
WDR82-binding long non-coding RNA IncEry controls mouse erythroid differentiation and maturation
}

Shangda Yang ${ }^{1,2 \#}$, Guohuan Sun ${ }^{1,2 \#}$, Peng Wu ${ }^{1,2 \#}$, Cong Chen ${ }^{3 \#}$, Yijin Kuang ${ }^{4}$, Zhaofeng Zheng ${ }^{1,2}$, Yicheng $\mathrm{He}^{1,2}$, Quan $\mathrm{Gu}^{1,2}$, Ting $\mathrm{Lu}^{1}$, Caiying Zhu ${ }^{1,2}$, Fengjiao Wang ${ }^{1,2}$, Fanglin Gou $^{3}$, Zining Yang ${ }^{1,2}$, Xiangnan Zhao ${ }^{1}$, Shiru Yuan ${ }^{1,2}$, Liu Yang ${ }^{1,2}$, Shihong Lu ${ }^{1,2}$, Yapu Li ${ }^{1,2}$, Xue Lv ${ }^{1,2}$, Fang Dong ${ }^{1,2}$, Yanni Ma ${ }^{5}$, Jia $\mathrm{Yu}^{5}$, Lai Guan $\mathrm{Ng}^{6}$, Lihong Shi ${ }^{1,2}$, Jing $\mathrm{Liu}^{4^{\star}}$, Hui Cheng ${ }^{1,2^{*}}$, and Tao Cheng ${ }^{1,2^{*}}$

${ }^{1}$ State Key Laboratory of Experimental Hematology, National Clinical Research Center for Blood Diseases, Institute of Hematology \& Blood Diseases Hospital, Chinese Academy of Medical Sciences \& Peking Union Medical College, Tianjin, China

${ }^{2}$ Center for Stem Cell Medicine, Department of Stem Cell \& Regenerative Medicine, Chinese Academy of Medical Sciences, Tianjin, China

${ }^{3}$ Department of Cell Biology, Tianjin Medical University, Tianjin, China

${ }^{4}$ Molecular Biology Research Center, Center for Medical Genetics, Hunan Province Key Laboratory of Basic and Applied Hematology, School of Life Sciences, Central South University, Changsha, China

${ }^{5}$ State Key Laboratory of Medical Molecular Biology, Key Laboratory of RNA Regulation and Hematopoiesis, Department of Biochemistry and Molecular Biology, Institute of Basic Medical Sciences, Chinese Academy of Medical Sciences, School of Basic Medicine Peking Union Medical College, Beijing, China

${ }^{6}$ Singapore Immunology Network, Agency for Science, Technology and Research, Biopolis, 138648 Singapore

"These authors contributed equally to this work.

*Correspondence: Tao Cheng (chengtao@ihcams.ac.cn), Hui Cheng (chenghui@ihcams.ac.cn) and Jing Liu (liujing2@sklmg.edu.cn) 


\begin{abstract}
Hematopoietic differentiation is controlled by both genetic and epigenetic regulators. Long non-coding RNAs (IncRNAs) have been demonstrated to be important for normal hematopoiesis, but their function in erythropoiesis needs to be further explored. We profiled the transcriptomes of 16 murine hematopoietic cell populations by deep RNA-sequencing and identified a novel IncRNA, Gm15915, that was highly expressed in erythroid-related progenitors and erythrocytes. For this reason, we named it IncEry. We also identified a novel IncEry isoform, which was also the principal transcript that has not been reported before. LncEry depletion impaired erythropoiesis, indicating the important role of the IncRNA in regulating erythroid differentiation and maturation. Mechanistically, we found that IncEry interacted with WD repeat-containing protein 82 (WDR82) to promote the transcription of Klf1 and globin genes and thus control the early and late stages of erythropoiesis, respectively. These findings identified IncEry as an important player in the transcriptional regulation of erythropoiesis.
\end{abstract}




\section{INTRODUCTION}

Hematopoietic stem cells (HSCs) are multipotent precursors with the capacity to self-renew and differentiate into all mature blood cell types ${ }^{1-4}$. During hematopoietic differentiation, long-term HSCs (LT-HSCs) differentiate into multiple blood cellular components ${ }^{5}$, including short-term HSCs (ST-HSCs), multipotent progenitor cells (MPPs), committed progenitor cells, and mature blood cells ${ }^{6,7}$. Hematopoiesis is tightly regulated by various regulatory elements, including non-coding RNAs (ncRNAs), to maintain normal biological processes ${ }^{8,9}$.

In mammals, approximately two-thirds of genomic DNA is pervasively transcribed ${ }^{10}$, while most genomic DNA is transcribed into ncRNAs, suggesting that RNA-based regulatory mechanisms might be involved in the complex developmental processes of eukaryotes ${ }^{11,12}$. These ncRNAs can be divided into two main types: small ncRNAs and long non-coding RNAs (IncRNAs). LncRNAs are defined as transcripts of >200 nucleotides with no apparent open reading frames ${ }^{9,13,14}$. Numerous functional IncRNAs have been discovered, including those that have a vital role in mediating hematopoiesis. For example, the oncofetal IncRNA gene H19 controls the balance between HSC quiescence and activation by regulating Igf2-Igf1r pathway activation ${ }^{15}$, promoting pre-HSC and HSC specification via the demethylation of hematopoietic transcription factors such as Runx1 and Spi $1^{16}$, or by participating in tumorigenesis ${ }^{17}$. LncHSC-1 and IncHSC-2 regulate the differentiation of myeloid and T cells, respectively ${ }^{4}$, while Inc-DC regulates monocyte-derived DC differentiation through STAT3 binding ${ }^{18}$. Erythropoiesis is also regulated by IncRNAs ${ }^{19-24}$, for example, IncRNA-EPS ${ }^{25}$ regulates the terminal differentiation of erythroid cells by promoting erythroid progenitor survival; IncRNA UCA1 controls erythropoiesis at the proerythroblast stage through the regulation of heme metabolism ${ }^{26}$; and $I n c-E C 1^{27}$ and $I n c-E C 6^{28}$ regulate erythroblast enucleation. Yet, despite such advances in our knowledge IncRNA identification, the function of most IncRNAs in erythropoiesis regulation remains largely unknown.

Erythroid Krüppel-like factor (EKLF; KLF1) ${ }^{29}$ is a zinc-finger hematopoietic transcription factor that plays a global role in regulating the activation of genes in different stages of erythropoiesis ${ }^{30,31}$. The selective expression of Klf1 promotes erythropoiesis and represses megakaryopoiesis ${ }^{32,33}$. During maturation, nucleated erythrocytes (NuEs) shed their nucleus and progressively gain erythroid characteristics, changing from nucleated erythrocytes to reticulocytes and ultimately mature blood cells. These mature blood cells are regulated by hemoglobins, which, if dysfunctional, can induce hemoglobinopathies such as $\beta$-thalassemia or sickle cell anemia ${ }^{34}$. In differentiated erythroid cells, the remote regulatory sequences of the $\alpha$-globin gene recruit polymerase II and the pre-initiation 
complex, then bind to transcription factors located in the promoter region to activate a-globin transcription ${ }^{35}$. Despite the depth of our understanding, we still need to ascertain the complexities of the transcriptional mechanisms regulating Klf1 and globin expression.

While many functional IncRNAs are recognized hematopoiesis mediators, the IncRNAs that regulate erythroid differentiation need clarification. Thus, we aimed to search for previously unidentified functional $\operatorname{IncRNA}(\mathrm{s})$ that play a role in erythroid differentiation by employing high-throughput RNA sequencing (RNA-seq) approaches. We further investigated the mechanisms of IncRNA in the regulation of erythropoiesis by promoting the transcriptional activation of KLF1 and globin genes at different stages of erythropoiesis.

\section{RESULTS}

\section{Gm15915 is highly expressed in an erythroid lineage}

Previous studies have confirmed the involvement of IncRNAs in many biological processes, including lineage differentiation ${ }^{4,36-38}$. To identify novel IncRNAs with biological relevance, we isolated 16 hematopoietic cell subsets from the bone marrow (BM) of C57BL/6 mice by fluorescence-activated cell sorting (FACS). These cell types included LT-HSCs, ST-HSCs, MPPs, common lymphoid progenitors (CLPS), common myeloid progenitors (CMPs), granulocyte-macrophage progenitors (GMPs), and megakaryocyte-erythroid progenitors (MEPs). We also isolated 10 mature lineage cell subsets: NK cells, B cells, CD4-T cells, CD8-T cells, monocytes, macrophages, granulocytes, megakaryocytes, and NuEs. Through RNA-seq, we identified 2,250 IncRNAs and 13,168 protein-coding genes. Thus, just a handful of highly expressed IncRNAs constituted the hematopoietic cell landscape (Figure 1A, Supplementary Table 1). Consistent with previous studies ${ }^{15}$, we found that the classical IncRNA H19 was highly expressed in LT-HSCs (Figure 1A), illustrating the validity of our approach.

To gain insights into the expression of IncRNAs in erythropoiesis, we decided to focus on the IncRNAs specifically expressed in MEPs, of which Gm15915 was the most highly expressed (Figure 1A). Gm15915 was also highly expressed in LT-HSCs, CMPs, and NuEs (Figure 1B, upper panel). Gm15915 levels positively correlated with the expression of erythroid lineage-development-associated genes, such as Gata1, KIf1, Tal1, and Car1 (Figure 1B, lower panel), suggesting Gm15915 is an erythroid-lineage-specific IncRNA (Figure 1C). Hence, Gm15915 was named "IncEry". To investigate IncEry further, we reanalyzed its expression in hematopoietic cells using a dataset from a previous study ${ }^{39}$. IncEry was highly expressed in LT-HSCs, ST-HSCs, CMPs, MEPs, and NuEs (Figure S1A), confirming our findings. In addition, by analyzing single-cell RNA-seq data from a previous study ${ }^{2}$, we found IncEry to be highly expressed in the MEP population (Figure 
S1B). Next, the expressions of IncEry in CMPs, GMPs, and MEPs were compared to determine any correlation with cell fate and found that IncEry expression increased as CMPs differentiated into MEPs but not GMPs (Figure S1C).

To further investigate IncEry function, we performed unsupervised hierarchical clustering on the expression levels of protein-coding and IncRNA genes. We defined 10 clusters and hypothesized that the genes expressed within the same cluster might have similar functions (Figure S1D). The genes in Cluster 9 were highly expressed in MEPs and NuEs (Figure 1D). We found known erythropoiesis-associated genes, such as Gata1, Klf1, Tal1, and Car1, and IncEry in Cluster 3. Finally, Gene Ontology (GO) enrichment analysis showed that the coding genes in Cluster 3 were significantly enriched for erythrocyte differentiation, supporting the functional role of IncEry in erythroid differentiation (Figure $1 \mathrm{E})$. Taken together, our data indicate that IncEry is potentially an important regulator of erythroid differentiation.

\section{LncEry is a bona fide long non-coding RNA}

To gain insights into the molecular characteristics of IncEry, we performed 5' and 3' rapid amplification of CDNA ends (RACE) PCR of MEP clones, followed by Sanger sequencing, to identifying IncEry transcript isoforms (Figure 2A). Two isoforms (isoform-1: NONMMUG004428.1 and isoform-2: NONMMUG0048.2) are annotated in the NONCODE database, and we discovered a third isoform, isoform-3, which shares four incomplete exons (exons 2-4, and 6 of isoform-1) with the other two (Figure 2B).

After analyzing the detailed sequence information (Figure S2A) and the RNA-seq read coverage of the IncEry gene in MEPs, we found IncEry isoform-3 to be the principal transcript (Figure 2B). Specifically, $\sim 79.5 \%$ and $\sim 20.5 \%$ of IncEry was expressed as isoform-3 and isoform-1/2, respectively. Similarly, $76 \%$ of IncEry in mouse erythroleukemia cell line (MEL) was expressed as isoform-3 (Figure 2C and Figure S2B, C). Next, we examined the coding capacity of isoform-3. Full-length isoform-3 was inserted into the eukaryotic expression vector pcDNA3.1 with N-terminal start codon ATG and C-terminal Flag tag in all three reading frames, and the results confirmed the non-protein-coding characteristics of isoform-3 (Figure 2D).

The functions of most IncRNAs are restricted to their subcellular localization ${ }^{40-42}$. We thus performed RNAscope assays to identify the location of IncEry in MEL cells. Nearly $90 \%$ of IncEry molecules localized to the nucleus (Figure 2E), which was confirmed by subcellular fractionation assay followed by quantitative PCR (qPCR) (Figure 2F). We presumed that the nuclear location of this IncRNA indicated its involvement in transcriptional regulation ${ }^{43}$. 
To prove this hypothesis, we transfected MEL cells with small interfering (si)RNAs targeting IncEry and analyzed the expression of the genes found within 1 Mbp upstream and downstream of the IncEry locus on chromosome 10. IncEry downregulation not only affected the expression levels of adjacent genes, such as Ntn4 and Ccdc38, but also influenced (to some extent) the expression of genes located $>100 \mathrm{Kbps}$ distant, e.g., Hal and Lta4h (Figure S2D). We thus speculated that IncEry has transcription regulatory capacity.

\section{Erythroid differentiation is impaired upon IncEry depletion in HSPCs}

To determine the functional impact of IncEry on HSPCs, we transduced cKit ${ }^{+}$cells with a lentivirus carrying GFP-fused IncEry short hairpin (sh)RNA, then performed in vitro colony forming unit (CFU) and in vivo transplantation assays (Figure S3A). First, we confirmed that the two shRNA constructs exhibited high-knockdown efficiencies at the mRNA level (Figure S3B). The CFU assays showed that IncEry-knockdown decreased the number of CFU-GM colonies by $32 \%$ on average and more potently decreased the number of BFU-E and CFU-GEMM colonies (74\% and $66 \%$ on average, respectively) (Figure S3C). In addition, colony sizes were significantly decreased upon IncEry knockdown (Figure S3D). These results are consistent with our hypothesis that IncEry is involved in erythroid differentiation.

Next, we infected donor cells with IncEry-shRNA-carrying lentiviruses, and after $48 \mathrm{~h}$ of culture, achieved transduction efficiencies of approximately $94 \%, 84 \%$, and $40 \%$ for control, shRNA-1, and shRNA-2, respectively (Figure S3E). We then transplanted the transduced cells (without sorting) into lethally irradiated mouse recipients (Figure S3A); 21 days after transplantation, two recipients from the IncEry shRNA-1 group died, and the remaining three mice in the IncEry shRNA-1 group showed pale paws and were moribund, indicative of severe anemia. The recipients of the IncEry shRNA-1 and IncEry shRNA-2 had decreased numbers of white blood cells (WBCs) and red blood cells (RBCs) (Figure $\mathrm{S} 3 \mathrm{~F}$ ), as well as very low hemoglobin levels compared with the controls (Figure S3F). Finally, the IncEry knockdown animals showed a decrease in the percentage of $\mathrm{GFP}^{+}$cells in the peripheral blood (PB) and BM (Figure S3G and $\mathrm{S} 3 \mathrm{H}$ ) and in the percentage of reticulocytes and RBCs in the GFP ${ }^{+}$cells within the BM (Figure S3I). We thus considered that IncEry is involved in erythroid differentiation from HSPCs.

\section{Erythroid differentiation is impaired in IncEry $\Delta / \Delta$ mice}

To study the function of IncEry in erythroid differentiation, we generated IncEry ${ }^{\mathrm{A} / \mathrm{fl}}$ (flox/flox) mice (Figure S4A). Then, we generated Mx1-Cre; IncEry ${ }^{f / f l}(\Delta / \Delta)$ mice, in which IncEry deletion could be induced by poly I:C administration ${ }^{44,45}$. We analyzed the expression of 
IncEry isoforms in the BM cells of both IncEryfl/fl and $\Delta / \Delta$ mouse strains: excision of exons $1 / 2$ of IncEry isoform-3 strongly decreased the expression of isoform-3 as well as the other two IncEry isoforms (Figure 3A).

To monitor the effects of IncEry on the hematopoietic system, we analyzed BM cells from flox/flox and $\Delta / \Delta$ mice by flow cytometry. We found that the $\Delta / \Delta$ mice had normal BM cellularity but an increased percentage of myeloid cells (Figure 3B and Figure S4B) and a decreased percentage of reticulocytes (Retic-E) and RBCs in BM and Ter119 ${ }^{+}$cells $^{46,47}$ (Figure 3C and Figure S4C-E). These findings are consistent with those of our knockdown assay (Figure S3I). In this case, however, it seemed that complete loss of IncEry might have impaired the terminal differentiation of erythropoiesis. To investigate how IncEry is involved in terminal erythroid differentiation, we analyzed several parameters in the PB of flox/flox and $\Delta / \Delta$ mice (Figure $3 \mathrm{C}$ ). We found that knocking out IncEry reduced the number of RBCs and Retic-Es as well as the concentration of hemoglobin (HGB) (Figure 3D) but had no effect on WBCs in $\Delta / \Delta$ mice.

Next, we examined the effects of knocking out IncEry on HSPCs. The percentage of most HSPC subsets (including LT-HSCs, ST-HSCs, MPP2, MPP3/4, CMPs, MEPs, and MKPs) decreased in $\Delta / \Delta$ mice compared with their littermate controls (Figures $3 \mathrm{E}, 3 \mathrm{~F}$ and Figure $\mathrm{S} 4 \mathrm{~F})$. Furthermore, the percentages of preMegE, CFU-E/Pro-E, and PreGM were significantly reduced in the knockout animals (Figure 3F), whereas CLPs and GMPs were minimally affected (Figure 3F and Figure S4G). Loss of IncEry apparently impairs the differentiation of erythroid lineage cells.

To examine the function of IncEry in erythropoiesis, we compared the colony-forming ability of $\mathrm{BM}$ cells from flox/flox and $\Delta / \Delta$ mice during in vitro culture in complete methylcellulose based medium. We found that the colony numbers of BFU-E, CFU-G, and CFU-GEMM from $\Delta / \Delta$ mice were lower than those from their littermate controls (Figure $3 G$ and Figure $\mathrm{S} 4 \mathrm{H}$ ). We then cultured BM or MEP cells in methylcellulose-based medium with erythropoietin (EPO) and established a BFU-E colony assay; the number and size of BFU-E colonies again decreased in $\Delta / \Delta$ compared to flox/flox mice (Figure $3 \mathrm{H}$ and Figure S4I). Finally, we cultured MEP cells sorted from flox/flox or $\Delta / \Delta$ mice in methylcellulose-based medium with EPO to support the optimal growth of CFU-E colonies. The colony number decreased for $\Delta / \Delta$ MEP cells (Figure 3l), indicating that loss of IncEry isoform-3 not only affects terminal differentiation during erythropoiesis but also reduces the growth of erythroid progenitor cells. 
To directly assess the effect of IncEry on the regenerative function of HSCs in vivo, we transplanted BM cells from flox/flox and $\Delta / \Delta$ mice (CD45.2) accompanied with competitor cells into irradiated recipients (CD45.1) (Figure 3J). The BM cells from $\Delta / \Delta$ mice had a lower reconstitution capacity than cells from control mice (71.1\% vs. 85.4\%) (Figure 3K), but donor cells from the two groups gave rise to the same level of myeloid (Mac- $1^{+}$) and lymphoid $\left(\mathrm{CD}^{+}\right.$and $\left.\mathrm{B} 200^{+}\right)$lineages (Figure $3 \mathrm{~L}$ ). The frequency of LT-HSCs in $\Delta / \Delta$ mice was approximately 2- to 3-fold lower than in the control mice (Figure 3E and S4G), and $\mathrm{BM}$ cells from $\Delta / \Delta$ mice showed a 2.3 -fold reduction in donor-cell engraftment 16 weeks after transplantation (Figure 3M). These data might explain the decreased level of engraftment observed in recipients when unseparated BM cells were transplanted. Together, these results demonstrated that loss of IncEry decreased the growth of MEPs, which ultimately led to suppressed erythroid differentiation and decreased RBC production. Interestingly, although IncEry deletion decreased the number of HSCs, the functions of the individual HSCs were unaffected.

We generated an additional IncEry ${ }^{f / f t}$ mouse (flox/flox-2) model by inserting loxP sites around exons 2-6 of IncEry isoform-1 using CRISPR/Cas9 technology (Figure S5A). Then, we generated Mx1-Cre; IncEry ${ }^{\mathrm{fl} / \mathrm{fl}}$ mice $(\Delta / \Delta-2)$. After poly I:C induction, the knockout efficiency of LncEry was confirmed by qPCR (Figure S5B). The deficits of erythroid differentiation and maturation were also observed in the new $\Delta / \Delta-2$ mice (Figure S5C-D). These results further confirmed the function of IncEry in regulating erythropoiesis.

\section{LncEry deletion decreases KIf1 expression in MEP cells}

To gain mechanistic insights into the function of IncEry in erythropoiesis, we isolated MEP cells from flox/flox and $\Delta / \Delta$ mice and performed RNA-seq analysis (Figure $4 \mathrm{~A}$ ). Compared with the flox/flox group, 3,256 genes were differentially expressed genes (DEGs), 1,054 of which were downregulated. To explore the changes in chromatin accessibility, we performed transposase-accessible chromatin (ATAC)-seq and observed 7,926 differential peaks (Figure 4B), and 4,599 peaks of accessibility were decreased upon deletion of IncEry in MEP cells. Notably, an integrative analysis with RNA-seq and ATAC-seq data revealed a significant correlation between downregulated genes and decreased accessibility, and we identified 421 overlapping genes (Figure S6A). In addition, GO enrichment analysis showed that the overlapping downregulated genes were enriched for erythrocyte differentiation and some metabolism-related terms. We then examined the ROS levels, mitochondrial membrane potential, and glucose uptake of MEP cells (Figure $\mathrm{S} 6 \mathrm{C}$ ), and the results indicated that the function of IncEry in erythropoiesis regulation does not depend on metabolic changes. 
The nuclear location of a IncRNA might be suggestive of its function in transcriptional regulation ${ }^{48,54}$. To understand the mechanisms of IncEry transcriptional regulation, we sought to determine the binding sites for IncEry in the genome through chromatin isolation by RNA purification sequencing (ChIRP-seq) ${ }^{4,48,49}$. We performed the ChIRP assay on MEL cells and confirmed the identities of the isolated RNAs by qPCR, and $\sim 17 \%$ of IncEry RNA was pulled down (Figure S6D). In the sequencing analysis, we identified $\sim 1,786$ IncEry binding sites when compared with input. When we analyzed the locations of these binding sites in the genome; 85\% were located in promoter regions (Figure 4C) and were mainly concentrated within a region $1 \mathrm{Kbp}$ from the transcriptional start sites (TSS) (Figure S6E). Some of these results support our theory of a role of IncEry in transcriptional regulation.

To further explore the transcriptional regulatory function of IncEry in MEP cells, we performed cleavage under targets and tagmentation (Cut\&Tag) assays ${ }^{50}$ on IncEry-deficient MEP cells using an antibody against the histone H3K4me3 (which is associated with transcriptional activation ${ }^{51}$ ), followed by sequencing (Figure S6F). Then, we performed integrative analysis of the downregulated genes from RNA-seq, ATAC-seq, ChIRP-seq, and H3K4me3 Cut\&Tag data to ascertain the directly regulated target genes, and 203 overlapping genes were identified (Figure 4D and Supplementary Table 2). GO enrichment analysis showed that these target genes were enriched in erythrocyte homeostasis- and differentiation-related terms (Figure 4E). In addition, the peaks of $\mathrm{H} 3 \mathrm{~K} 4 \mathrm{me} 3$ and H3K27ac at the cis-regulate region (CRR) of the Klf1 gene were decreased upon IncEry deletion (Figure 4F, S6G-H). Importantly, IncEry could bind to the CRR of KIf1, and deletion of IncEry decreased KIf1 expression (Figure $4 \mathrm{G}$ and S6I), chromatin accessibility, and the transcriptional active stage of KIf1 CRR (Figure 4F), suggesting that IncEry participates in the transcriptional regulation of KIf1 in MEP cells. LncEry deletion also significantly decreased the expression of other erythrocyte-differentiation-related genes: Fech, Ldb1, Rhd, and Car2 (Figure 4G). In addition, gene set enrichment analysis (GSEA) revealed that deletion of LncEry in MEP cells reduced the enrichment of KLF1-target genes (Figure S6H). Together, these results indicate that IncEry regulates the transcription of $K l f 1$ to affect the early stage of erythropoiesis.

\section{LncEry regulates late-stage erythropoiesis by promoting globin gene expression}

To further explore the function of IncEry in the late stage of erythropoiesis, we transfected MEL cells with siRNAs targeting IncEry and performed RNA-seq analysis. Compared with the control group, 117 and 134 DEGs after IncEry knockdown with siRNA-1 and siRNA-2, respectively (Figure 5A). Of these DEGs, 75 overlapping genes were downregulated (Figure 5B and Supplementary Table 3). When we conducted enrichment analysis of 
these 75 genes with the Metascape online tool, strikingly, the top most enriched term was erythrocyte homeostasis (Figure $5 \mathrm{C}$ ).

We then performed qPCR to analyze the expression of several DEGs: IncEry knockdown significantly decreased the expression of erythrocyte-homeostasis- and differentiation-related genes, such as Hba-a1, Hba-a2 (downregulated in siEry2 parts) (two variants of $\alpha$-globin), $H b b-b 1, H b b-b 2$ (two variants of $\beta$-globin), Alas2, and Rhag in MEL cells (Figure S7A). Furthermore, IncEry knockdown significantly reduced the protein levels of $\alpha$ - and $\beta$-globin (Figure S7B). We also examined DEGs in NuEs sorted from the $\mathrm{BM}$ of flox/flox and $\Delta / \Delta$ mice, and IncEry knockout decreased mRNA expression and protein levels of $\alpha$ - and $\beta$-globin (Figure 5D-E). Unlike in MEPs, IncEry knockout did not reduce the expression of Klf1 in NuE cells (Figure 5D-E), and the DEGs in MEL cells were also not enriched in Klf1-target genes (Figure S7C). These results indicate that IncEry participates in erythropoiesis regulation at different stages and using different mechanisms.

To further explore the role of IncEry in regulating DEGs at the transcriptional level, we performed chromatin immunoprecipitation (ChIP) assays in IncEry-deficient cells using antibodies against Ser5-phosphorylated RNA polymerase II (Pol II-S5p) and histone H3K4me3, followed by sequencing. We then compared the peaks enriched by Pol II-S5p and histone H3K4me3 of the gene promoters in control and IncEry-depleted cells. Interestingly, we found four downregulated DEGs in our RNA-seq dataset (Hbb-b1, Hba-a1, Hbb-b2, and Btg2) that overlapped in the Pol II-S5p and H3K4me3 ChIP-sequencing datasets (Figure 5F and Figure S7D). Consistently, the Pol II-S5p and H3K4me3 enrichment peaks of globin gene cis-regulated regions, such as Hba-a1, $H b a-a 2, H b b-b 1$, and Hbb-b2, sharply declined upon IncEry knockdown (Figure 5G and Figure S7E), and the results of the qChIP assays verified these findings (Figure $5 \mathrm{H}$ ). We thus concluded that IncEry depletion affects the transcription of globin genes in the later stage of erythropoiesis.

\section{LncEry is physically associated with Wdr82}

LncRNAs are usually associated with numerous cellular functions, most of which require interactions with one or more RNA-binding proteins ${ }^{52,53}$. To determine whether IncEry acts alone or in concert with other proteins in the different stages of erythropoiesis, we performed RNA-pulldown assays (Figure S8A) followed by silver staining and mass spectrometry to identify IncEry isoform-3 interaction partners in MEPs sorted from the BM of wildtype mice and MEL cells. Then, we performed integrative analysis and found 11 common interacting partners in the two types of cells (Figure 6A-B, S8B, and 
Supplementary Tables 4 and 5). Interestingly, we identified two transcription regulators, WD repeat-containing protein 82 (WDR82) and DEAD-box helicase 5 (DDX5), as likely IncEry binding partners, which may explain some of the mechanisms involved in regulating the DEGs of IncEry-depleted MEP and NuE cells at the transcriptional level. Western blot analyses confirmed the interactions between IncEry and its binding partners (Figure 6C).

To explore the functional impact of Wdr82 and Ddx5 on HSPCs, we transduced cKit ${ }^{+}$cells with a lentivirus carrying GFP-fused Wdr82 or $D d \times 5$ shRNAs and performed in vitro colony assays (Figure S8C and S8D). The results showed that Wdr82 knockdown decreased the numbers of CFU-G, CFU-M, and BFU-E, whereas Ddx5 knockdown did not decrease BFU-E numbers (Figure S8C and S8D). The knockdown efficiency was confirmed by western blotting (Figure S8E). Then, we cultured Wdr82 knockdown cKit ${ }^{+}$cells in M3436 methylcellulose-based medium with EPO and established a BFU-E colony assay. The colony number and size of the BFU-E colonies decreased in Wdr82-knockdown cells (Figure S8F). However, when we cultured the cKit ${ }^{+}$cells in vitro for about 1 week after they were transduced with Wdr82 shRNA, Wdr82 knockdown was seen to decrease the

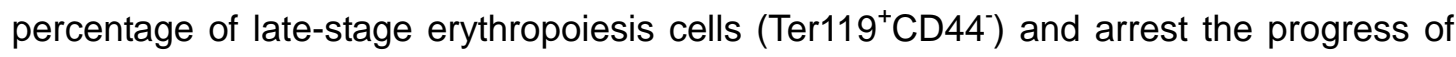
erythropoiesis (Figure S8G). These results suggest that Wdr82, with similar phenotypes to IncEry, was more likely to participate in erythropoiesis with IncEry than Ddx5. Following this, we were interested in exploring the function of IncEry interaction with Wdr82 in regulating the early and late stages of erythropoiesis. When we examined the interaction between IncEry isoform-1 or isoform-3 and Wdr82, we found no evidence of transcriptional specificity (Figure 6D). Consistent with these findings, RNA immunoprecipitation (RIP) assays further confirmed this interaction between all IncEry isoforms and Wdr82 (Figure 6E).

We were then intrigued to identify the binding sites underlying the IncEry-Wdr82 interaction. To do so, we generated IncEry isoform-3 truncation mutants according to the isoform's main minimum free energy (MFE) stem-loop regions, which we predicted using RNAfold WebServer tools (http://rna.tbi.univie.ac.at) (Figure 6F, upper panel). In vitro RNA-pulldown assays showed that the interaction was primarily dependent on the IncEry C-terminal loop regions (Figure 6F). We also generated two IncEry fragments that are shared by all three isoforms: exons 2-4 and 6 of IncEry isoform-1. The results of the binding assay revealed that IncEry mainly interacted with Wdr82 through the last exon transcript (exon 6) (Figure 6G). Next, we conducted surface plasmon resonance assays using the GE Healthcare Biacore 3000 platform to examine the binding kinetics of IncEry and Wdr82. Indeed, the last exon region IncEry-P5 (exon 6) shared by each of the three 
IncEry isoforms directly interacted with Wdr82 that was purified from MEL cell lines (Figure $\mathrm{S} 8 \mathrm{H}$ ) with a KD value of $38.2 \mathrm{nM}$ (Figure $6 \mathrm{H}$ ). Finally, we performed co-localization assays combining RNAscope with immunofluorescent staining followed by fluorescent confocal microscopy. We observed that IncEry mainly co-localized with Wdr82 in the nuclei of MEP and MEL cells (Figure $6 \mathrm{I}$ and Figure S8I). All IncEry isoforms physically associated with Wdr82 in the nuclei of MEP and MEL cells. We thus proposed a hypothesis that the IncEry-Wdr82 complex serves to regulate transcription in these cells.

\section{LncEry-Wdr82 regulates the transcriptional activation of Klf1 in MEP cells}

As IncEry was found to be associated with Wdr82 (Figure 6), we further explored the molecular mechanisms of the role of IncEry and Wdr82 in MEP cells by performing Cut\&Tag assays on IncEry-deficient MEP cells using an antibody against Wdr82. The results showed that IncEry deletion decreased chromatin accessibility and the binding of Wdr82 at CRR around KIf1 gene body as well as the binding of Wdr82 at whole genome region (Figure 7A and S9A). Therefore, we speculated that IncEry can physically interact and functionally coordinate with Wdr82 to regulate the transcription of Klf1. To test this, we established two pGL3-luciferase reporters containing CRRs, as shown in Figure 7A, then performed reporter assays with pGL3-luciferase reporters containing KIf1 CRRs or mutant CRRs (without the main LncEry binding site) co-transfected into 293T cells together with IncEry, Wdr82, or both, as well as the Renila luciferase vector for normalization (Figure 7B and S9B). The assays showed that overexpression of IncEry or co-expression of IncEry and Wdr82 enhanced the activity of the Klf1-CRR reporter, but not the Klf1-CRRs-mt reporter (Figure 7B). These results suggest that IncEry coordinates with Wdr82 to regulate the transcription of Klf1 in early erythroid differentiation.

\section{LncEry-Wdr82 regulates the transcriptional activation of globin genes}

In the late stage of erythropoiesis, IncEry depletion decreased the transcription of globin genes. We found that IncEry was located at the CRRs of Hba-a1 and Hba-a2 (Figure 7C) but not the genomic regions of $H b b-b 1$ and $H b b-b 2$ (Figure S9C). Furthermore, using qPCR, we detected IncEry binding on the CRRs of globin genes (Figure 7D and Figure $\mathrm{S} 9 \mathrm{C})$. However, we were unable to detect IncEry binding on Hbb-b1 and Hbb-b2 CRRs, possibly because it has lower affinity for these promoters or because cofactors are required for IncEry binding.

Because IncEry associated with Wdr82 and directly bound to the CRRs of globin genes in MEL cells (Figure 6C and 6D), we speculated that IncEry physically interacts and functionally coordinates with Wdr82 to regulate globin gene transcription in late erythropoiesis. To test this, MEL cells were co-transfected pGL3-luciferase reporters 
containing Hba-a1, Hba-a2, Hbb-b1, or Hbb-b2 promoters (Figure 7E) along with IncEry, Wdr82, or both, or the Renila luciferase vector. Reporter assays showed that overexpression of either IncEry or Wdr82 resulted in a significant increase in Hba-a1, Hba-a2, Hbb-b1, and Hbb-b2 reporter activity. Co-expression of IncEry and Wdr82 enhanced $\mathrm{Hba}-\mathrm{a} 1$ and $\mathrm{Hba}-\mathrm{a} 2$ (but not $\mathrm{Hbb}-\mathrm{b} 1$ or $\mathrm{Hbb}-\mathrm{b} 2$ ) reporter activity further, which was perhaps because reporter activity was saturated (Figure 7E). Flag-Wdr82 expression was confirmed by western blotting (Figure S9D).

To investigate whether the effects of IncEry on the transcriptional activation of globin genes were associated with Wdr82, we transfected Flag-Wdr82 plasmids into IncEry-deficient MEL cells. Subsequent luciferase reporter assays showed that IncEry depletion inhibited Hba-a1, Hba-a2, Hbb-b1, and Hbb-b2 reporter activities, but this inhibition was rescued, at least in part, upon Flag-Wdr82 overexpression (Figure 7F and Figure S9E-H). Similarly, Wdr82 depletion inhibited the four globin gene reporter activities, and this inhibition was rescued by IncEry overexpression (Figure 7F and Figure S9E-H).

We confirmed the effects of IncEry and Wdr82 on globin gene transcription at the protein level, and again, IncEry depletion decreased the protein expression of $\alpha$-globin and $\beta$-globin, and Wdr82 overexpression partially rescued the effect (Figure 7G, left panel) and vice versa (Figure 7G, right panel). Interestingly, IncEry depletion decreased the level of $\mathrm{H} 3 \mathrm{~K} 4 \mathrm{me} 3$, which could be rescued by overexpression of Wdr82, and vice versa (Figure $7 \mathrm{G}$ and Figure S9I). These results led us to propose that IncEry might participate in the Set1A/Wdr82 complex to affect $\mathrm{H} 3 \mathrm{~K} 4 \mathrm{me} 3$ levels and, as a result, regulate the transcriptional activation of globin genes.

To strengthen the above hypothesis, our final analyses examined the effects of IncEry on Wdr82 and Set1A recruitment to globin gene CRRs using qChIP assay. To this end, we immunoprecipitated soluble chromatin from control or IncEry-depleted MEL cells using antibodies against Wdr82 or Set1A, and then performed qPCR analysis to identify the precipitated DNA. IncEry depletion specifically decreased Wdr82 and Set1A enrichment on the CRRs of globin genes, but not the two other components of the Set1A/Wdr82 complex Ash2l and RbBP5 ${ }^{54}$ (Figure $7 \mathrm{H}$ and S9J). These findings might be due to the low affinity of Ash2l and RbBp5 antibodies for ChIP samples (Figure $7 \mathrm{H}$ and Figure S9J). Collectively, these results support the hypothesis that IncEry recruits Wdr82 and stabilizes the Set1A/Wdr82 complex located on the CRRs of globin genes (Figure 8). 


\section{DISCUSSION}

Because a number of IncRNAs have been shown to be important for hematopoiesis, in this study, we aimed to investigate the importance of these regulatory factors in erythropoiesis, about which much less is known. We found a novel functional IncRNA, Gm15915 (IncEry), that is specifically and highly expressed in MEPs and NuEs, and we annotated a new and highly expressed IncEry isoform localized in the nucleus. LncEry depletion decreased Klf1 and globin gene expression in erythroid progenitors and mature cells, respectively, and therefore impaired murine erythroid cell differentiation and maturation. Mechanistically, IncEry together with Wdr82 facilitates the binding of the histone $\mathrm{H} 3 \mathrm{~K} 4 \mathrm{me} 3$ to the CRRs of Klf1 and globin genes, which in turn, regulates the early and late stages of erythropoiesis (Figure 8).

Erythroid differentiation is regulated at multiple levels to ensure the proper generation of mature cells under multiple physiological conditions ${ }^{27}$. Numerous functional IncRNAs that regulate cellular processes, such as cell development, differentiation, division, survival, and death, have been identified in recent years ${ }^{55,56}$. The nuclear localization of IncEry suggests that it might participate in regulating gene expression by modulating certain nuclear events such as epigenetic modifications, transcription, or mRNA splicing ${ }^{57}$. Wdr82 is a unique subunit of the Set1 A (KMT2F) histone H3-Lys4 methyltransferase complex and can recruit the Set1A complex to the transcription start sites of target genes and bind to Ser 5 phosphorylated RNA polymerase II to promote transcriptional activation ${ }^{58-60}$. Recruitment of the KMT2 complex to specific chromatin regions by IncRNAs has been reported $^{61}$, which supports our hypothesis that IncEry interacts with Wdr82 and participates in transcriptional activation at CRRs. Furthermore, Wdr82 depletion results in dysfunction of the Set1A/Set1B complex, affecting H3K4me3 status and inhibiting the transcriptional activation of Pou5f1, thereby preventing early embryonic development ${ }^{62}$. Whether IncEry also participates in embryonic development with Wdr82 needs further exploration.

For transcriptional regulation, recruitment of the mixed lineage leukemia (MLL) complex to specific chromatin regions not only depends on the presence of plant homeodomains but also IncRNAs; for example a long intergenic non-coding RNA, HOTTIP, guides WDR5 to chromatin to recruit the MLL complex ${ }^{63,64}$. As a member of the Set1A complex, Wdr82 has affinity for the IncEry $\mathrm{C}$ terminus, which is a common region of all IncEry isoforms. IncEry can generally target CRRs of Klf1 and globin genes, thus we speculated that binding of the Wdr82/Set1 A complex to the genome is likely to partially depend on IncEry. According to previous reports, Wdr82 promotes Set1A-dependent H3K4 trimethylation ${ }^{54}$ to participate in transcriptional activation. Even so, IncEry depletion decreased enrichment of 
Wdr82 and the level of H3K4me3 in the CRRs of Klf1 and globin genes, which supports our hypothesis that IncEry directs the Set1A/Wdr82 complex to the CRRs of Klf1 and globin genes in MEP and MEL cells and stabilizes its location. We thus believe we have identified a new and important regulator associated with the Wdr82/Set1A complex that promotes the transcriptional activation of Klf1 and globin genes in early- and late-stage erythropoiesis, respectively.

Most IncRNAs show little conservation between species and exhibit a rapid evolutionary turnover. Indeed, we did not find IncEry in human cells, yet there are two annotated IncRNAs (ENST00000552603.1 and ENST0000055074.1) similar to IncEry located anti-sense of Ntn4. However, we did not manage to show these IncRNAs to have the same function or expression pattern as IncEry (data not shown). Despite this, we cannot exclude the possibility that other functional IncRNAs bind to the Wdr82/Set1A complex to participate in related biological processes.

In conclusion, we have identified a potential component of the Wdr82/Set1 A complex that participates in the transcriptional regulation of KIf1 and globin genes and, by extension, the early and late stages of erythroid differentiation to regulate erythropoiesis. Whether IncRNAs together with Wdr82 are viable targets for manipulating other processes and their clinical application need to be addressed in additional research. 


\section{METHODS}

Mice

C57BL/6J and B6.SJL mice were purchased from the animal facility of the State Key Laboratory of Experimental Hematology (SKLEH, Tianjin, China). IncEry ${ }^{\text {flox } /+}$ mice were generated by Beijing Biocytogen Co.,Ltd. All animal procedures were performed in compliance with the animal care guidelines approved by the Institutional Animal Care and Use Committees of the SKLEH and the Institute of Hematology.

\section{Antibodies and reagents}

The following antibodies were used in this study: FLAG (F1804, 1:2000 for Western blot), $\beta$-actin (A1978, 1:10,000 for WB) from Sigma; histone H3 (ab1791, 1:10,000 for WB), RNA polymerase II (phospho S5) (ab5408, 1:200 for ChIP), H3K4me3 (ab8580, 1:200 for ChIP and Cut\&Tag), and H3K27ac (ab6002, 1:200 for Cut\&Tag) from Abcam; Wdr82 (99715, 1:1000 for WB, 1:100 for Cut\&Tag), DDX5 (9877T, 1:1,000 for WB), and RBBP5 (13171S, 1:200 for ChIP) from Cell Signaling Technology; PCBP2 (NBP2-19715, 1:1,000 for WB) from Novusbio; PTBP1 (32-4800, 1:1,000 for WB) from Invitrogen; a-globin (14537-1-AP, 1:2,000 for WB) from Proteintech; EKLF (OM184222, 1:1,000 for WB) and B-globin (OM256195, 1:1,000 for WB) from OmnimAbs; ASH2L (A11278, 1:200 for ChIP) from Abclonal. Anti-FLAG M2 affinity gel (A2220), 3 × FLAG peptide (F4799), doxycycline (D9891), and 2',7'-dichlorofluorescin diacetate (DCF-DA) (35845) were purchased from Sigma. Guinea pig anti-rabbit IgG (Heavy \& Light Chain) antibody (ABIN101961, 1:100 for Cut\&Tag) was from Antibodies Online. Tetramethylrhodamine methyl ester (TMRE) (I34361) and 2-NDBG [2-(N-(7-Nitrobenz-2-oxa-1,3-diazol-4-yl)Amino)-2-Deoxyglucose] (N13195) were purchased from Invitrogen. Recombinant murine SCF (250-03), murine IL-3 (213-13) and Recombinant Human EPO (100-64) were obtained from PeproTech.

\section{ROS levels, mitochondrial membrane potential and glucose uptake analysis}

MEP cells from flox/flox or $\Delta / \Delta$ mice were labeled with surface markers, and after 30 min of incubation at $4^{\circ} \mathrm{C}$, the cells were washed with $1 \mathrm{~mL}$ staining buffer. Then, the cells were stained with DCFH-DA, TMRE or 2-NDBG for 20 minutes at $37^{\circ} \mathrm{C}$ with shaking, and washed with $2 \mathrm{~mL}$ cold staining buffer. Finally, the cells were immediately analyzed by flow cytometry.

\section{Plasmids and viral production}

The FLAG-tagged IncEry isoforms or truncation mutants were expressed using a pcDNA3.1 vector. FLAG-tagged Wdr82 was inserted into a pLenti-Tight-Puro vector. Klf1, Hba-a1, Hba-a2, Hbb-b1, and Hbb-b2 CRRs and luciferase were ligated into pGL3-luciferase vectors. The IncEry, Wdr82, and Ddx5 LV-shRNA-GFP lentiviruses were 
produced by GeneChem (Shanghai, China). For lentiviral production, the target plasmid was transfected together with PSPAX2 and pMD2G into 293T cell lines using Lipofectamine 2000. The supernatant was harvested after 48 and $72 \mathrm{~h}$ of culture and concentrated using an Amicon filter (100K NMWL, Millipore).

\section{Cell culture}

MEL and HEK293T cells were purchased from the American Type Culture Collection (Manassas, VA) and cultured in RPMI1640 (A10491-01, Gibco) or DMEM (SH30243.01, Hyclone) medium with $10 \%$ fetal bovine serum (FBS) respectively. BM cells were obtained from C57BL/6 mice and cultured in SFEM (09650) medium (STEM CELL). Cells that allow protein expression under doxycycline treatment were created using two steps. First, cells were infected with a lentivirus carrying rtTA and selected using neomycin. The established rtTA cells were subsequently infected with a virus expressing pLenti-Tight-Puro vector that encodes Wdr82 and selected using puromycin. All of the cells integrated with rtTA were cultured in Tet-Approved FBS and RPMI1640. All cells were authenticated by examination of their morphology and growth characteristics and were confirmed to be mycoplasma-free.

\section{Western blotting}

Western blotting was performed as described previously ${ }^{65}$. In brief, cellular extracts were harvested from cells and resuspended in $5 \times$ SDS-PAGE loading buffer. The boiled protein samples were then subjected to SDS-PAGE followed by immunoblotting with the appropriate primary antibodies and secondary antibodies.

\section{RACE assays}

5' and 3' RACE reactions were performed to isolate full-length IncEry from the total RNA of MEP cells using the 5'- and 3'-Full RACE Kits (TaKaRa, Dalian, China) in accordance with the manufacturer's instructions. Primers used for RACE are listed in Supplementary Table 6.

\section{Flow cytometry}

For cell-sorting experiments using mouse MEP cells, $\mathrm{cKit}^{+}$cells were enriched before flow cytometry using cKit magnetic beads (Miltenyi Biotec). The cells were subsequently stained with a lineage cocktail and cKit (eBioscience, 17-1171-82, 1:200), Sca-1 (eBioscience, 25-5981-82, 1:200), CD34 (eBioscience, 13-0341-82, 1:100), and CD16/CD32 (eBioscience, 45-0161-82, 1:200) antibodies. The lineage cocktail included Gr-1 (Biolegend, 108424, 1:400), Mac-1 (Biolegend, 101226, 1:400), B220 (Biolegend, 103224, 1:400), CD4 (Biolegend, 100414, 1:400), CD8 (Biolegend, 100714, 1:400), CD3 
(Biolegend, 100330, 1:400), and Ter-119 (Biolegend, 116223, 1:400). DAPI (D9542, 1 $\mathrm{mg} / \mathrm{mL}$, Sigma-Aldrich) was used to exclude dead cells. For the HSPC analysis, nucleated BM cells were stained with lineage-specific antibodies against Sca-1, cKit, CD34, CD16/CD32, and CD127 (Biolegend, 135040, 1:400). For PreMegE and MPP analyses, nucleated BM cells were stained with lineage cocktail, Sca-1, cKit, CD16/CD32, CD41 (eBioscience, 46-0411-82, 1:400), CD150 (Biolegend, 115904, 1:400), CD48 (Biolegend, 103432, 1:400), and CD105 (Biolegend, 120409, 1:400). The lineage cocktail included CD3, CD4, CD8, B220, Gr-1, Ter119, and Mac-1. For Pro-E analysis, nucleated BM cells were stained with CD3, B220, Mac-1, Ter119, and CD44 (eBioscience, 45-0441-82, 1:200). A modified LSR II flow cytometer with four lasers (355 nm, $488 \mathrm{~nm}, 561 \mathrm{~nm}$, and $633 \mathrm{~nm}$ ) was used for the analysis, and an Aria III flow cytometer with four lasers (375, 488, 561, and $633 \mathrm{~nm}$ ) was used for sorting. The analyses were performed using FACSDiVa and FlowJo (Tree Star) software.

\section{Colony-forming assays}

Murine BM cells were cultured in M3434 complete methylcellulose-based medium (03434, StemCell Technologies) for 10-14 days to generate BFU-E, CFU-G, CFU-M, CFU-GM, and CFU-GEMM colonies. Murine BM or sorted MEP cells were cultured in M3436 medium (03436, StemCell Technologies) for $48 \mathrm{~h}$ to generate CFU-E colonies. Murine-sorted MEP cells were cultured in M3334 medium (03334, StemCell Technologies) for 10-14 days to generate BFU-E colonies.

\section{Transplantation}

For IncEry knockdown assay, GFP-fused control or IncEry shRNA lentiviruses were transduced into B6.SJL (CD45.1 $1^{+}$) mouse cKit ${ }^{+}$HSPCs, and $4 \times 10^{5}$ transduced cells $\left(\mathrm{CD} 45.1^{+} \mathrm{GFP}^{+}\right)$were transplanted together with $1.5 \times 10^{5} \mathrm{CD} 45.2^{+} \mathrm{BM}$ cells into lethally irradiated C57BL/6J (CD45.2) recipients and repopulation was measured monthly. For competitive BM transplantation, $5 \times 10^{5} \mathrm{BM}$ cells of flox/flox or $\Delta / \Delta$ mice, were transplanted into lethally irradiated (9.5 Gy) B6.SJL recipient mice in competition with $2.5 \times 10^{5}$ CD45. $1^{+} \mathrm{BM}$ cells and repopulation was measured.

\section{RNA pulldown and mass spectrometry analyses}

Substrate RNAs were synthesized by in vitro transcription using a T7 RNA production system (Promega, P1300) in accordance with the manufacturer's instructions. The 3'-end desthiobiotin-labeled RNA probes used in the RNA pulldown were generated using an RNA 3' End Desthiobiotinylation Kit (20163, Thermo Scientific) following the manufacturer's instructions. RNA pulldown was performed using a Magnetic RNA-Protein Pull-Down Kit (20164, Thermo Scientific). In brief, 3'-end desthiobiotin-labeled RNA 
probes were captured by streptavidin magnetic beads and mixed with MEP or MEL cell extracts (containing $1 \mathrm{mg}$ of protein) in IP lysis buffer (87787, Thermo Scientific) in protein-RNA-binding buffer and incubated for 30-60 $\mathrm{min}$ at $4^{\circ} \mathrm{C}$ with agitation or rotation. After general washing, the retrieved proteins were detected by western blotting or subjected to silver staining and LC-MS/MS analysis (Supplementary Tables 4 and 5).

\section{Nano-HPLC-MS/MS analysis of IncEry-binding proteins}

To identify proteins associated with IncEry, LC-MS/MS analysis was performed on an Orbitrap Q Exactive mass spectrometer. Tryptic peptides were separated by reverse-phase liquid chromatography on an easy-nLC 1000 system (Thermo Fisher Scientific) and directly sprayed into a Q Exactive Plus mass spectrometer (Thermo Fisher Scientific). Mass spectrometry analysis was carried out in data-dependent mode with an automatic switch between a full MS and an MS/MS scan on the Orbitrap. For the full MS scan, the automatic gain control target was 1 e6 and the scan ranged from 300 to 1800 with a resolution of 70,000 . The 10 most intense peaks with a charge state $\geq 2$ were selected for fragmentation by high-energy collision dissociation with a normalized collision energy of $27 \%$. The MS2 spectra were acquired with 17,500 resolutions. All MS/MS spectra were searched against the Uniport-Human protein sequence database using Mascot 2.2. Peptide sequences were searched using trypsin specificity while allowing for a maximum of two missed cleavages. Cys carbamidomethylation was specified as a fixed modification, and oxidation of methionine was fixed as a variable modification. The peptide mass tolerance was set at $\pm 20 \mathrm{ppm}$, and the fragment mass tolerance was set at $\pm 0.1 \mathrm{Da}$.

\section{RNA immunoprecipitation (RIP)}

RIP assays were performed using a Magna Nuclear RIP Kit from Merck Millipore (17-10520, Millipore) with $5 \mu \mathrm{g} \operatorname{lgG}$ or anti-Wdr82 antibody in accordance with the manufacturer's instructions. The eluted RNAs were purified using TRIzol LS regent (Life Technology). We then performed qPCR analysis with the indicated primers (Supplementary Table 6).

\section{Quantitative PCR (qPCR)}

Total cellular RNA was isolated with TRIzol reagent (Invitrogen) and used for first-strand cDNA synthesis via the Reverse Transcription System (Roche). Quantitation of all gene transcripts was made by qPCR using a Power SYBR Green PCR Master Mix (Roche) and Thermo Quant Studio 5 sequence detection system (Thermo) with the expression of ACTB as the internal control. The primers used are listed in Supplementary Table 6. 


\section{RNAscope and Immunofluorescence assays}

50,000 cells were centrifuged onto slides on $800 \mathrm{~g}$ for $5 \mathrm{~min}$. Cells were fixed with $4 \%$ paraformaldehyde for $15 \mathrm{~min}$ and dehydrated in 50\%, 70\%, and 100\% ethanol for $2 \mathrm{~min}$ each. After incubating with hydrogen peroxide and proteinase IV, the samples were treated using the RNAscope multiplex fluorescent V2 assay kit (323100, ACD) and incubated with the specific probes (Mm-Gm15915-O2: 555551; Negative Control DapB: 310043 and Positive Control Mm-Ppib: 313911 from ACD) and Cy3 dye (designed by ACD) in accordance with the manufacturer's instructions. Before staining with DAPI, the samples were incubated with the appropriate primary and secondary antibodies coupled to AlexaFluor 488 (Invitrogen) following the instructions provided by ACD. Confocal images were captured using a Spinning Disk Confocal Microscope system (UltraVIEW VOX) with a $\times 100$ oil objective. To avoid bleed-through effects in the double-staining experiment, each dye was scanned independently in multi-tracking mode.

\section{Recombinant protein purification}

Lysates from MEL cells stably expressing FLAG-Wdr82 were prepared by incubating the cells in lysis buffer [300 mM NaCl, 1\% NP-40, 0.1\% SDS, 0.5\% sodium-deoxycholate, and $50 \mathrm{mM}$ Tris- $\mathrm{HCl}$ ( $\mathrm{pH}$ 8.0)] containing a protease inhibitor cocktail (Roche). Anti-FLAG immunoaffinity columns were prepared using anti-FLAG M2 affinity gel (Sigma) following the manufacturer's instructions. Cell lysates were applied to an equilibrated FLAG column of 200- $\mu \mathrm{l}$ bed volume to allow for protein adsorption to the column resin. After binding, the column was extensively washed with high-salt solution for $5 \mathrm{~min}$ five times each [300 mM $\mathrm{NaCl}, 150 \mathrm{mM} \mathrm{KCl}, 1 \% \mathrm{NP}-40,0.1 \% \mathrm{SDS}, 0.5 \%$ sodium-deoxycholate, and $50 \mathrm{mM}$ Tris. $\mathrm{HCl}(\mathrm{pH}$ 8.0)]. A FLAG peptide (Sigma) was applied to the column to elute the FLAG protein complex, as recommended by the manufacturer. The eluents were collected and visualized by SDS-PAGE followed by Coomassie blue staining. The purified protein was used in subsequent molecular interaction assays.

\section{Surface plasmon resonance (SPR)}

Sensorgrams of IncEry-P5 binding to Wdr82 were measured by SPR technology using a Biacore 3000 instrument (GE Healthcare). The flowing-phase RNA IncEry-P5 was synthesized by in vitro transcription using a T7 RNA production system (Promega, P1300) in accordance with the manufacturer's instructions. The substrate-phase Wdr82 proteins were purified from MEL cells and immobilized on a CM5 sensor chip (BIAcore) with the following procedure. All experiments were carried out using HBS-EP (10 mM HEPES pH $7.4,150 \mathrm{mM} \mathrm{NaCl}, 3.4 \mathrm{mM}$ EDTA, and $0.005 \%$ surfactant P20) as the running buffer at a constant flow rate of $30 \mu \mathrm{l} / \mathrm{min}$ at $25^{\circ} \mathrm{C}$. The $\mathrm{Wdr} 82$ protein was diluted in $10 \mathrm{mM}$ sodium acetate buffer $(\mathrm{pH} 4.0)$ to a final concentration of $2.5 \mu \mathrm{M}$ and covalently immobilized on 
the hydrophilic carboxymethylated dextran matrix of the CM5 sensor chip using a standard primary amine coupling procedure. LncEry-P5 was dissolved in the running buffer to concentrations ranging from $36.7 \mathrm{nM}$ to $294 \mathrm{nM}$. All data were analyzed by BIA evaluation software, and the sensorgrams were processed by automatic correction for nonspecific bulk refractive index effects. Kinetic analyses of IncEry-P5/Wdr82 binding were performed based on the 1:1 Langmuir binding fit model in accordance with the procedures in the software manual.

\section{RNA interference}

All siRNA transfections were performed using Lipofectamine RNAiMAX (Invitrogen) following the manufacturer's recommendations. The final concentration of the siRNA molecules was $10 \mathrm{nM}$, and the cells were harvested $72 \mathrm{~h}$ or $96 \mathrm{~h}$ after transfection, depending on the experiment. Control, IncEry, and Wdr82 siRNAs were chemically synthesized by Sigma (Shanghai, China). The shRNAs against IncEry, Wdr82, or Ddx5 in lentivirus U6-MSX-IRES vectors were purchased from GeneChem. The siRNA and shRNA sequences are provided in Supplementary Table 6.

\section{RNA-sequencing and analysis}

RNA-Seq included three parts of experiment samples. First, a total of 16 hematopoietic cell populations were sorted from the BM of C57BL/6 mice. Second, MEP cells were sorted from the BM of flox/flox or $\Delta / \Delta$ mice. Third, MEL cells were transfected with control or IncEry siRNAs. Total RNA was isolated using the RNeasy (Qiagen) purification kit. Libraries were prepared using an Illumina RNA library preparation TruSeq PE kit. High-throughput RNA-Seq was performed on an Illumina Xten sequencer (paired-end 150 bp sequencing). Clean reads were filtered by removing reads including adapters, reads including poly- $\mathrm{N}$ and low-quality reads from raw data. All the following analyses were based on clean data. Clean data were firstly aligned to the mouse genome (GRCm38) with GENCODE M16 gene annotation using HISAT2 (v2.1.0) ${ }^{66}$. DEG analyses were conducted using Cufflinks and Cuffdiff (v2.2.1) software ${ }^{67}$. Unsupervised hierarchical clustering was conducted using pheatmap R package. Genes with the q-value less than 0.05 in the Cuffdiff results were considered to be significantly different genes.

\section{Assay for Transposase-Accessible Chromatin sequencing}

ATAC-seq was performed as described previously ${ }^{68}$, In brief, 50,000 MEP cells sorted from BM of flox/flox or $\Delta / \Delta$ mouse cells were lysed in lysis buffer $(10 \mathrm{mM}$ Tris-HCl, $\mathrm{PH} 7.4$, $10 \mathrm{mM} \mathrm{NaCl}, 3 \mathrm{mM} \mathrm{MgCl}, 0.1 \%$ (v/v) IGPAL CA-630) for 10 min on ice. After centrifuged on $500 \mathrm{~g}$ for $5 \mathrm{~min}$, the obtained nuclei were added to $50 \mu \mathrm{L}$ transposition reaction buffer (offered by Vazyme TD501-01) followed with incubation at $37^{\circ} \mathrm{C}$ for $30 \mathrm{~min}$. After 
tagmentation, VAHTS DNA Clean Beads were used to stop the reaction, and DNA was purified for final library construction (TruePrep DNA Library Prep Kit V2 for Illumina) before paired-end high-throughput sequencing using HiSeq XTe. Clean reads were aligned to the mouse genome (GRCm38) using BWA package, and peaks were called using MACS2 package $(\mathrm{v} 2.2 .5)^{69}$ with a false discovery rate (FDR) cutoff of 0.05 .

\section{Cleavage under targets and tagmentation}

Cut\&Tag experiments were performed as described previously ${ }^{50}$ together with the Hyperactive TM In-Situ ChIP Library Prep Kit for Illumina from Vazyme (TD902-01). MEP cells sorted from the $\mathrm{BM}$ of flox/flox or $\Delta / \Delta$ mice cells were captured by ConA beads and incubated with primary and secondary antibodies in Antibody buffer and Dig-wash Buffer, respectively, for the time indicated in the manufacturer's instructions. The cells were then incubated with Hyperactive pA-Tn5 Transposon and fragmented in Tagmentation Buffer at $37^{\circ} \mathrm{C}$ for $1 \mathrm{~h}$. The extracted DNA fragments were analyzed by high-throughput sequencing. Clean reads were aligned to the mouse genome (GRCm38) using the Bowtie2 package ${ }^{70}$, and Peaks were detected using MACS2 callpeak (v2.2.5) ${ }^{69}$ with an FDR cutoff of 0.05 .

\section{Chromatin immunoprecipitation sequencing}

ChIP-seq was performed as described previously ${ }^{71}$. In brief, $\sim 10$ million cells were crosslinked with $1 \%$ formaldehyde for $10 \mathrm{~min}$ at room temperature and quenched by adding glycine to a final concentration of $125 \mathrm{mM}$ for $5 \mathrm{~min}$. The fixed cells were resuspended in SDS lysis buffer [1\% SDS, $5 \mathrm{mM}$ EDTA, and $50 \mathrm{mM}$ Tris- $\mathrm{HCl}(\mathrm{pH}$ 8.1)] in the presence of protease inhibitors and subjected to sonication (Bioruptor, Diagenode) to generate chromatin fragments of $\sim 300$ bp in length. For immunoprecipitation, after dilution, the chromatin was incubated with control or specific antibodies $(3 \mu \mathrm{g})$ overnight at $4^{\circ} \mathrm{C}$ with constant rotation; $50 \mu \mathrm{L}$ of $50 \%$ (vol/vol) protein $\mathrm{G}$ magnetic beads was then added, and the incubation was continued for an additional $2 \mathrm{~h}$. The beads were washed with the following buffers: TSE I, TSE II, buffer III, and Tris-EDTA buffer as described previously ${ }^{71}$. Then the cross-links were removed from the pulled-down chromatin complex together with the input sample by incubation at $65^{\circ} \mathrm{C}$ for $2 \mathrm{~h}$ in elution buffer. The eluted DNA was purified using a PCR purification kit (Qiagen) and analyzed by qPCR using the primers detailed in Supplementary Table 6 or by high-throughput sequencing. Clean reads were aligned to the mouse genome $(\mathrm{GRCm} 38)$ using the Bowtie2 package ${ }^{70}$. ChIP-seq peaks were detected using MACS2 callpeak (v2.2.5) ${ }^{69}$ with a minimum FDR cutoff of 0.05.,

\section{Chromatin Isolation by RNA purification sequencing}

ChIRP-seq was performed as described previously ${ }^{48}$. Eluted DNA was purified using a PCR purification kit (Qiagen) and analyzed by qPCR using the qChIP primers described in 
Supplementary Table 6 or by high-throughput sequencing. The processing of sequencing data was the same as that for the ChIP-seq data described above. Peaks were annotated using the ChIPseeker R package (v1.24.0 $)^{72}$.

\section{Luciferase reporter assay}

The modulation of globin gene promoter activity by IncEry was analyzed by luciferase assay. A pGL3 construct carrying the globin gene CRRs sequence was obtained by PCR enrichment using the primers (Supplementary Table 6), and the Klf1 CRRs sequence was synthesized. Luciferase reporter activity was measured using the Dual Luciferase Assay System (E1910, ProMega). Relative luciferase activity was normalized to Renila luciferase and control vector luciferase reporter activity.

\section{Statistical analyses}

Data from biological triplicate experiments are presented, and the data represent the means \pm S.D. A two-tailed unpaired Student's t-test was used to compare two groups of data. Analysis of variance (ANOVA) with Bonferroni's correction was used to compare multiple groups of data. A $P$ value $<0.05$ was considered statistically significant. All statistical analyses were performed in SPSS 20.0 software, and graphs were generated by GraphPad Prism 8.0 software.

\section{Data availability}

RNA-seq datasets of 12 hematopoietic cell populations were deposited in the NCBI GEO (GSE142216). Other sequencing data have been deposited in the NCBI SRA (PRJNA647682).

\section{Acknowledgments}

The authors would like to thank all lab members for their assistance with the experiments. They thank Dr. Yiran Ren for providing help on SPR assays. This work was supported by grants from the Ministry of Science and Technology of China (2017YFA0103400, 2020YFE0203000, 2016YFA0100600), the National Natural Science Foundation of China (81922002, 81730006, 81861148029, 81890990, 81870086, 81900113, 81890993, 81900117, 81920108004), the CAMS Initiative for Innovative Medicine (2017-I2M-3-009, 2016-I2M-1-017), the CAMS Fundamental Research Funds for Central Research Institutes (2019RC310003), Distinguished Young Scholars of Tianjin (19JCJQJC63400).

\section{Author contributions}

SD.Y., GH.S. and C.C., designed and performed the experiments, analyzed the data, and wrote the paper. P. W. performed bioinformatic analyses. YJ.K., ZF.Z., YC.H., Q.G., FJ.W., 
FL.G., ZN.Y., X.L., XN.Z, and SH.L. helped with mouse experiments. T.L., CY.Z., and L.Y. helped with bioinformatic analyses. YP.L. helped with experimental design. YN.M., J.Y., SR.Y., F.D., LH.S., and LG.N. assisted with the manuscript. J.L., H.C., and T.C. proposed the study, designed the experiments, interpreted the results, wrote the paper, and oversaw the research project.

\section{Competing interests}

The authors have no competing interests to declare. 


\section{REFERENCES}

1. Wilson, A. et al. Hematopoietic stem cells reversibly switch from dormancy to self-renewal during homeostasis and repair. Cell 135, 1118-29 (2008).

2. Nestorowa, S. et al. A single-cell resolution map of mouse hematopoietic stem and progenitor cell differentiation. Blood 128, e20-31 (2016).

3. Busch, K. et al. Fundamental properties of unperturbed haematopoiesis from stem cells in vivo. Nature 518, 542-6 (2015).

4. Luo, M. et al. Long non-coding RNAs control hematopoietic stem cell function. Cell Stem Cell 16, 426-38 (2015)

5. Orkin, S.H. \& Zon, L.I. Hematopoiesis: an evolving paradigm for stem cell biology. Cell 132, 631-44 (2008).

6. Nakamura, Y. et al. Isolation and characterization of endosteal niche cell populations that regulate hematopoietic stem cells. Blood 116, 1422-32 (2010).

7. Nilsson, L. et al. Isolation and characterization of hematopoietic progenitor/stem cells in 5q-deleted myelodysplastic syndromes: evidence for involvement at the hematopoietic stem cell level. Blood 96, 2012-21 (2000).

8. Delas, M.J. et al. IncRNA Spehd Regulates Hematopoietic Stem and Progenitor Cells and Is Required for Multilineage Differentiation. Cell Rep 27, 719-729 e6 (2019).

9. Li, W., Ren, Y., Si, Y., Wang, F. \& Yu, J. Long non-coding RNAs in hematopoietic regulation. Cell Regen (Lond) 7, 27-32 (2018).

10. Ulitsky, I. \& Bartel, D.P. lincRNAs: genomics, evolution, and mechanisms. Cell 154, 26-46 (2013).

11. Fatica, A. \& Bozzoni, I. Long non-coding RNAs: new players in cell differentiation and development. Nat Rev Genet 15, 7-21 (2014).

12. Djebali, S. et al. Landscape of transcription in human cells. Nature 489, 101-8 (2012).

13. Clark, M.B. et al. Quantitative gene profiling of long noncoding RNAs with targeted RNA sequencing. Nat Methods 12, 339-42 (2015).

14. Mattick, J.S. \& Makunin, I.V. Non-coding RNA. Hum Mol Genet 15 Spec No 1, R17-29 (2006).

15. Venkatraman, A. et al. Maternal imprinting at the H19-lgf2 locus maintains adult haematopoietic stem cell quiescence. Nature 500, 345-9 (2013).

16. Zhou, J. et al. Combined Single-Cell Profiling of IncRNAs and Functional Screening Reveals that H19 Is Pivotal for Embryonic Hematopoietic Stem Cell Development. Cell Stem Cell 24, 285-298 e5 (2019).

17. Raveh, E., Matouk, I.J., Gilon, M. \& Hochberg, A. The H19 Long non-coding RNA in cancer initiation, progression and metastasis - a proposed unifying theory. Mol Cancer 14, 184 (2015).

18. Wang, P. et al. The STAT3-binding long noncoding RNA Inc-DC controls human dendritic cell differentiation. Science 344, 310-3 (2014).

19. Kulczynska, K. \& Siatecka, M. A regulatory function of long non-coding RNAs in red blood cell development. Acta Biochim Pol 63, 675-680 (2016).

20. Paralkar, V.R. et al. Lineage and species-specific long noncoding RNAs during erythro-megakaryocytic development. Blood 123, 1927-37 (2014).

21. An, X. et al. Global transcriptome analyses of human and murine terminal erythroid differentiation. Blood 123, 3466-77 (2014). 
22. Shi, L. et al. Developmental transcriptome analysis of human erythropoiesis. Hum Mol Genet 23, 4528-42 (2014).

23. Arriaga-Canon, C. et al. A long non-coding RNA promotes full activation of adult gene expression in the chicken $\alpha$-globin domain. Epigenetics 9, 173-81 (2014).

24. Xu, C. \& Shi, L. Long non-coding RNAs during normal erythropoiesis. Blood Science 1, 137-140 (2019).

25. Hu, W., Yuan, B., Flygare, J. \& Lodish, H.F. Long noncoding RNA-mediated anti-apoptotic activity in murine erythroid terminal differentiation. Genes Dev 25, 2573-8 (2011).

26. Liu, J. et al. Long non-coding RNA-dependent mechanism to regulate heme biosynthesis and erythrocyte development. Nat Commun 9, 4386 (2018).

27. Alvarez-Dominguez, J.R. et al. Global discovery of erythroid long noncoding RNAs reveals novel regulators of red cell maturation. Blood 123, 570-81 (2014).

28. Wang, C. et al. Shlnc-EC6 regulates murine erythroid enucleation by Rac1-PIP5K pathway. Dev Growth Differ 57, 466-73 (2015).

29. Miller, I.J. \& Bieker, J.J. A novel, erythroid cell-specific murine transcription factor that binds to the CACCC element and is related to the Krüppel family of nuclear proteins. Mol Cell Biol 13, 2776-86 (1993).

30. Gnanapragasam, M.N. \& Bieker, J.J. Orchestration of late events in erythropoiesis by KLF1/EKLF. Curr Opin Hematol 24, 183-190 (2017).

31. Mukherjee, K. et al. EKLF/KLF1 expression defines a unique macrophage subset during mouse erythropoiesis. Elife 10(2021).

32. Frontelo, P. et al. Novel role for EKLF in megakaryocyte lineage commitment. Blood 110, 3871-80 (2007).

33. Siatecka, M. \& Bieker, J.J. The multifunctional role of EKLF/KLF1 during erythropoiesis. Blood 118, 2044-54 (2011).

34. Stamatoyannopoulos, G. Control of globin gene expression during development and erythroid differentiation. Exp Hematol 33, 259-71 (2005).

35. Vernimmen, D., De Gobbi, M., Sloane-Stanley, J.A., Wood, W.G. \& Higgs, D.R. Long-range chromosomal interactions regulate the timing of the transition between poised and active gene expression. EMBO J 26, 2041-51 (2007).

36. Guttman, M. et al. lincRNAs act in the circuitry controlling pluripotency and differentiation. Nature 477, 295-300 (2011).

37. Klattenhoff, C.A. et al. Braveheart, a long noncoding RNA required for cardiovascular lineage commitment. Cell 152, 570-83 (2013).

38. Hung, T. et al. Extensive and coordinated transcription of noncoding RNAs within cell-cycle promoters. Nature Genetics 43, 621-U196 (2011).

39. Qian, P. et al. The Dlk1-Gt/2 Locus Preserves LT-HSC Function by Inhibiting the PI3K-mTOR Pathway to Restrict Mitochondrial Metabolism. Cell Stem Cell 18, 214-28 (2016).

40. Chen, L.L. Linking Long Noncoding RNA Localization and Function. Trends Biochem Sci 41, 761-772 (2016).

41. Batista, P.J. \& Chang, H.Y. Long noncoding RNAs: cellular address codes in development and disease. Cell 152, 1298-307 (2013).

42. Chen, L.L. \& Carmichael, G.G. Decoding the function of nuclear long non-coding RNAs. Curr Opin Cell Biol 22, 357-64 (2010). 
43. Carlevaro-Fita, J. \& Johnson, R. Global Positioning System: Understanding Long Noncoding RNAs through Subcellular Localization. Molecular Cell 73, 869-883 (2019).

44. Aliprantis, A.O. et al. NFATc1 in mice represses osteoprotegerin during osteoclastogenesis and dissociates systemic osteopenia from inflammation in cherubism. J Clin Invest 118, 3775-89 (2008).

45. Ruocco, M.G. et al. I\{kappa\}B kinase (IKK)\{beta\}, but not IKK\{alpha\}, is a critical mediator of osteoclast survival and is required for inflammation-induced bone loss. J Exp Med 201, 1677-87 (2005).

46. Liu, J. et al. Quantitative analysis of murine terminal erythroid differentiation in vivo: novel method to study normal and disordered erythropoiesis. Blood 121, e43-9 (2013).

47. Chen, K. et al. Resolving the distinct stages in erythroid differentiation based on dynamic changes in membrane protein expression during erythropoiesis. Proc Natl Acad Sci U S A 106, 17413-8 (2009).

48. Chu, C., Qu, K., Zhong, F.L., Artandi, S.E. \& Chang, H.Y. Genomic maps of long noncoding RNA occupancy reveal principles of RNA-chromatin interactions. Mol Cell 44, 667-78 (2011).

49. Engreitz, J.M. et al. The Xist IncRNA exploits three-dimensional genome architecture to spread across the $X$ chromosome. Science 341, 1237973 (2013).

50. Kaya-Okur, H.S. et al. CUT\&Tag for efficient epigenomic profiling of small samples and single cells. Nat Commun 10, 1930 (2019).

51. Ptashne, M. \& Gann, A. Transcriptional activation by recruitment. Nature 386, 569-577 (1997).

52. Cao, M., Zhao, J. \& Hu, G. Genome-wide methods for investigating long noncoding RNAs. Biomed Pharmacother 111, 395-401 (2019).

53. Ferre, F., Colantoni, A. \& Helmer-Citterich, M. Revealing protein-IncRNA interaction. Brief Bioinform 17, 106-16 (2016).

54. Wu, M. et al. Molecular regulation of H3K4 trimethylation by Wdr82, a component of human Set1/COMPASS. Mol Cell Biol 28, 7337-44 (2008).

55. Sabin, L.R., Delas, M.J. \& Hannon, G.J. Dogma derailed: the many influences of RNA on the genome. Mol Cell 49, 783-94 (2013).

56. Gallagher, P.G. Long noncoding RNAs in erythropoiesis. Blood 123, 465-6 (2014).

57. Sun, Q., Hao, Q. \& Prasanth, K.V. Nuclear Long Noncoding RNAs: Key Regulators of Gene Expression. Trends Genet 34, 142-157 (2018).

58. Rao, R.C. \& Dou, Y. Hijacked in cancer: the KMT2 (MLL) family of methyltransferases. Nat Rev Cancer 15, 334-46 (2015).

59. Deng, C. et al. USF1 and hSET1A mediated epigenetic modifications regulate lineage differentiation and HoxB4 transcription. PLoS Genet 9, e1003524 (2013).

60. Lee, J.H. \& Skalnik, D.G. CpG-binding protein (CXXC finger protein 1) is a component of the mammalian Set1 histone H3-Lys4 methyltransferase complex, the analogue of the yeast Set1/COMPASS complex. J Biol Chem 280, 41725-31 (2005).

61. Guo, C.J. et al. Distinct Processing of IncRNAs Contributes to Non-conserved Functions in Stem Cells. Cell 181, 621-636 e22 (2020).

62. $\mathrm{Bi}, \mathrm{Y}$. et al. WDR82, a key epigenetics-related factor, plays a crucial role in normal early embryonic development in mice. Biol Reprod 84, 756-64 (2011).

63. Yang, Y.W. et al. Essential role of IncRNA binding for WDR5 maintenance of active chromatin 
and embryonic stem cell pluripotency. Elife 3, e02046 (2014).

64. Wang, K.C. et al. A long noncoding RNA maintains active chromatin to coordinate homeotic gene expression. Nature 472, 120-4 (2011).

65. Yang, S. et al. USP52 acts as a deubiquitinase and promotes histone chaperone ASF1A stabilization. Nat Commun 9, 1285 (2018).

66. Kim, D., Langmead, B. \& Salzberg, S.L. HISAT: a fast spliced aligner with low memory requirements. Nat Methods 12, 357-60 (2015).

67. Trapnell, C. et al. Transcript assembly and quantification by RNA-Seq reveals unannotated transcripts and isoform switching during cell differentiation. Nat Biotechnol 28, 511-5 (2010).

68. Wang, Y. et al. Targeting of apoptosis gene loci by reprogramming factors leads to selective eradication of leukemia cells. Nat Commun 10, 5594 (2019).

69. Zhang, Y. et al. Model-based analysis of ChIP-Seq (MACS). Genome Biol 9, R137 (2008).

70. Langmead, B. \& Salzberg, S.L. Fast gapped-read alignment with Bowtie 2. Nat Methods 9, 357-9 (2012).

71. Shang, Z. et al. USP9X-mediated deubiquitination of B-cell CLL/lymphoma 9 potentiates Wnt signaling and promotes breast carcinogenesis. J Biol Chem 294, 9844-9857 (2019).

72. Yu, G., Wang, L.G. \& He, Q.Y. ChIPseeker: an R/Bioconductor package for ChIP peak annotation, comparison and visualization. Bioinformatics 31, 2382-3 (2015). 


\section{Figure Legends}

\section{Figure 1. Gm15915 is highly expressed in erythroid lineage}

(A) Heat map of IncRNA expression across 16 hematopoietic cell populations. Representative IncRNAs in different cell types are shown on the right. The top 10 IncRNAs are shown in Supplementary Table 1. (B) RNA-seq analysis of IncEry expression in the 16 hematopoietic cell populations (upper panel). Correlation analysis of IncEry and erythroid lineage related gene expression in the 16 hematopoietic cell populations (lower panel). Each bar represents normalized duplicate samples. (C) Schematic representation of the hematopoietic hierarchy showing the cell types used in this study; the intensity of the red color indicates the level of IncEry expression. (D) Line chart showing Cluster 9 gene expression in the 17 hematopoietic cell populations. (E) Gene enrichment analysis showing - $\log 10$ of the uncorrected $\mathrm{P}$-values on the $\mathrm{x}$-axis; darker shading corresponds to a greater amount of enriched genes in each term.

\section{Figure 2. LncEry is a bona fide long non-coding RNA}

(A) 5' and 3' RACE assays and gel electrophoresis analysis to detect IncEry transcripts in of MEP cells. (B) RNA-seq tracks at IncEry loci with different read numbers linked to the different exons of the IncEry isoforms. The shared exons of the different isoforms are marked by the red frame. LncEry isoform-1: NONMMOUG004428.1; IncEry isoform-2: NONMMUG004428.2. (C) Expression of different IncEry isoforms in MEP and MEL cells analyzed by qPCR. Error bars represent the mean \pm S.D. for biological triplicates. (D) Western blot showing that there was no expression of Flag-tagged IncEry isoform-3 in all three reading frames in MEL cells. Flag-tagged KLF4 was used as a positive control. Full-length IncEry isoform-3 was cloned into the eukaryotic expression vector pcDNA3.1(-) with an $\mathrm{N}$-terminal start codon ATG and a C-terminal Flag tag in all three reading frames; these plasmids were transfected into MEL cells separately and analyzed by western blotting.. (E) RNAscope and confocal microscopy analysis of IncEry subcellular localization in MEL cells. Ppib and DapB probes were used as positive and negative controls, respectively. The percentage of different IncEry subcellular localization points in $>50$ cells was calculated. Scale bar, $10 \mu \mathrm{m}$. (F) The IncEry fraction in the cytosol and nucleus. Gapdh and mU1 were used as markers of the cytosolic and nuclear RNA fractions, respectively. Each error bar represents the mean \pm S.D. of triplicate experiments. The separation efficiency of each cell component was determined by western blotting.

\section{Figure 3. Erythroid differentiation is impaired in IncEry $\Delta / \Delta$ mouse}

(A) qPCR analysis of IncEry isoform expression in flox/flox or $\Delta / \Delta$ bone marrow (BM) cells. (B-C) Percentage of indicated cell populations in flox/flox or $\Delta / \Delta$ mice BM. Each bar represents the mean \pm S.D. of triplicate experiments. ${ }^{*} P<0.05$, ${ }^{*} P<0.01$, one-way 
ANOVA. (D) Absolute numbers or concentrations of the indicated items in the peripheral blood of flox/flox or $\Delta / \Delta$ mice. (E-F) Percentage of indicated cell populations in the BM of flox/flox or $\Delta / \Delta$ mice. (G) $1 \times 10^{4} \mathrm{BM}$ cells from flox/flox or $\Delta / \Delta$ mice were cultured for 10-14 days in colony forming unit (CFU) assays in complete methylcellulose-based medium, and colony numbers were counted. (H) BFU-E CFU assays of $1 \times 10^{4}$ flox/flox or $\Delta / \Delta$ mice $B M$ cells cultured in methylcellulose-based medium with EPO cytokine supplementation for 10-14 days. Representative images from triplicate experiments are shown. (I) CFU-E colony assays of 500 flox/flox or $\Delta / \Delta$ mice MEP cells cultured in methylcellulose-based medium with EPO cytokine for $48 \mathrm{~h}$. Representative images from triplicate experiments are shown. Scale bar, $50 \mu \mathrm{m}$. (J) Experimental flow-chart of competitive transplantation; mice were treated with poly I:C $25 \mathrm{mg} / \mathrm{g}$ three times every other day before transplantation. (K) Percentage of $C D 45.2^{+}$cells in the peripheral blood (PB) of recipient $\left(C D 45.1^{+}\right)$mice. (L) Percentage of CD45.2 $2^{+}$cells in indicated populations 4 months after transplant. (M) Repopulating units $(R U)$ of donor cells calculated after 4 months reconstitution. Each bar represents the mean \pm S.D. for triplicate experiments. ${ }^{*} P$ $<0.05,{ }^{* *} \mathrm{P}<0.01$.

\section{Figure 4. LncEry deletion decreases Klf1 expression in MEP cells}

(A) Heat map of differentially expressed genes from RNA-seq of MEP cells sorted from BM of flox/flox or $\Delta / \Delta$ mice. (B) Heat map showing replication of samples from ATAC-seq of MEP cells sorted from BM of flox/flox or $\Delta / \Delta$ mice. (C) Distribution of IncEry-binding sites across the indicated intergenic or intragenic regions in MEL cells, as shown by chromatin isolation by ChIRP-seq. (D) Cut\&Tag sequence analysis of H3K4me3 in MEP cells sorted from $\mathrm{BM}$ of flox/flox or $\Delta / \Delta$ mice; the decreased peak genes under IncEry deletion overlapped with the downregulated genes detected by RNA-seq, downregulated peak genes from ATAC-seq and IncEry-binding peak genes from ChIRP-seq. (E) GO term analysis of the downregulated genes in (D), $-\log 10$ of the uncorrected P-value on the $x$ axis. (F) Visualization of RNA-seq, ATAC-seq, Cut\&Tag sequence of H3K4me3 and H3K27ac and ChIRP-seq peaks and predict cis-regulate elements in Klf1 regions with IGV software. (G) qPCR analysis of the indicated genes in MEP cells sorted from BM of flox/flox or $\Delta / \Delta$ mice. ${ }^{*} \mathrm{P}<0.05,{ }^{* *} \mathrm{P}<0.01$.

\section{Figure 5. LncEry regulates late stage of erythropoiesis by promoting globin gene expression}

(A) Heat map of differentially expressed genes in IncEry-siRNA-treated and control (CT) MEL cells according to RNA-seq. (B) The numbers of overlapping, downregulated, differentially expressed genes in IncEry-depleted and CT MEL cells using two different targeting siRNAs. (C) Gene enrichment analysis of downregulated genes with -log 10 plot 
of the uncorrected P-value on the x-axis; darker shading corresponds to a greater level of enriched genes in each term. (D) qPCR of indicated genes in NuE cells sorted from BM of flox/flox or $\Delta / \Delta$ mice. (E) Cellular extracts of NuE cells sorted from BM of flox/flox, $\Delta / \Delta$, flox/flox-2, or $\Delta / \Delta-2$ mice were prepared and analyzed by western blotting. $(F)$ MEL cells were transfected with control siRNA or IncEry siRNA. Soluble chromatin was collected for ChIP-seq analysis using antibodies against Pol II-S5p or H3K4me3; the decreased peak genes under IncEry knockdown overlapped with the downregulated genes detected by RNA-seq. (G) ChIP-seq trace showing Pol II-S5p and H3K4me3 binding of control or IncEry knockdown cells in relation to the indicated gene CRRs, visualized with IGV software. (H) qChIP of Pol II-S5p (left panel) or H3K4me3 (right panel) with primers covering the promoters of the indicated genes. Each bar represents the mean \pm S.D. for triplicate experiments. ${ }^{*} \mathrm{P}<0.05,{ }^{* *} \mathrm{P}<0.01$.

\section{Figure 6. LncEry is physically associated with Wdr82}

(A-B) RNA pulldown and mass spectrometry analyses of IncEry-interacting proteins. Whole-cell extracts from MEP (A) or MEL (B) were prepared and subjected to RNA pulldown using in vitro transcribed IncEry-3 or anti-IncEry-3 as the bait. After extensive washing, the bound proteins were eluted and visualized by silver staining on SDS-PAGE. The protein bands on the gel were recovered by trypsinization and analyzed by mass spectrometry. Detailed results from the mass spectrometric analysis are provided in Supplementary Tables 4 and 5. (C-D) RNA-pulldown-purified proteins retrieved using the indicated baits were analyzed by western blotting with antibodies against the indicated proteins. (E) Whole-cell lysates from MEL cells were immunoprecipitated with Wdr82 antibodies; purified RNA was analyzed by qPCR and agarose gel electrophoresis. (F) Schematic of minimum free energy (MFE) structure and the IncEry isoform-3 truncation mutant. RNA-pulldown-purified proteins retrieved by IncEry truncation mutant baits were analyzed by western blotting. (G) Schematic of the MFE structure and the IncEry truncation mutants. RNA pull-down purified proteins by IncEry truncation mutants baits were analyzed by western blotting with antibodies against the indicated proteins. $(H)$ Sensorgrams of IncEry-P5 binding to Wdr82, as measured by SPR technology on a Biacore 3000 instrument. Representative sensorgrams were obtained by injecting various concentrations $(0,36.7,73.4,147$, and $294 \mathrm{nM})$ of IncEry-P5 over Wdr82 immobilized on a CM5 sensor chip. (I) RNAscope and immunofluorescence assays using IncEry probes and Wdr82 antibodies, respectively, of MEP cells and analysis by confocal microscopy. Scale bar, $10 \mu \mathrm{m}$. Each bar represents the mean \pm S.D. for triplicate experiments. ${ }^{* *} \mathrm{P}<$ 0.01 . 
Figure 7. LncEry-Wdr82 regulates transcriptional activation of Klf1 and globin genes

(A) Visualization of ATAC-seq, Cut\&Tag for H3K4me3 and Wdr82, ChIRP-seq IncEry peaks, predict cis-regulate elements, and luciferase reporter clone CRRs in Klf1 gene regions with IGV software. (B) Schematic diagrams of the pGL3-CRRs-luciferase reporter constructs and relative luciferase activity was determined by sequential normalization to Renilla and pGL3-vector activity. (C) ChIRP-seq trace showing IncEry binding in relation to the indicated gene regions. Red boxes indicate the CRRs of globin genes (D) qPCR and agarose gel electrophoresis analysis of isolated chromatin sequences of indicated gene promoter regions. (E) Schematic diagrams of pGL3-CRRs-luciferase reporter constructs (upper panel). For reporter assays, MEL cells were co-transfected with pcDNA3.1-IncEry or Wdr82 or pcDNA3.1-IncEry and Wdr82 together with Renilla and globin gene CRRs luciferase. The relative luciferase activity was determined by sequential normalization to Renilla and pGL3-vector activity. (F) MEL cells were co-transfected with the indicated siRNAs or plasmids together with Renilla and the globin gene CRRs luciferase. The relative luciferase activity was determined by sequential normalization to Renilla and pGL3-vector activity. (G) MEL cells were co-transfected with the indicated siRNAs or plasmids and the cellular extracts were analyzed by western blotting with antibodies against the indicated proteins. $(H)$ MEL cells were transfected with control or IncEry siRNAs; the soluble chromatin was immunoprecipitated with antibodies against Wdr82 or Set1A and analyzed by qPCR with the indicated primers. The relative fold enrichment was determined by sequential normalization with the cycle threshold values of the input and control siRNA samples. Each bar represents the mean \pm S.D. for biological triplicate experiments. ${ }^{*} \mathrm{P}<0.05,{ }^{* *} \mathrm{P}<0.01$.

Figure 8. Mechanistic model of how IncEry combines with Wdr82 to participate in erythropoiesis regulation

Generally, the novel annotated IncRNA, IncEry, interacts with Wdr82 to control the location of the Set1A/Wdr82 complex and facilitate H3K4me3 binding to CRRs of target genes. In MEP cells, IncEry bind to the CRRs of Klf1 to recruit Wdr82 and elevate H3K4me3 levels at CRRs to promote Klf1 expression and coordinate early erythroid differentiation. In NuE, IncEry binds to CRRs of globin genes and combines with Wdr82 to promote transcription of $\mathrm{Hba-a1}, \mathrm{Hba}-\mathrm{a} 2, \mathrm{Hbb}-\mathrm{b} 1$, and $\mathrm{Hbb}-\mathrm{b} 2$, which promotes terminal erythropoiesis. In brief, IncEry combines with Wdr82 to promote Klf1 and globin gene expression to regulate the early and late stages of erythropoiesis, respectively. 
A

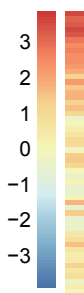

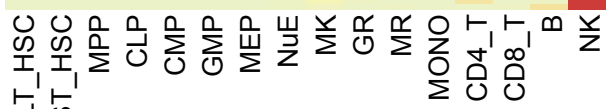

C

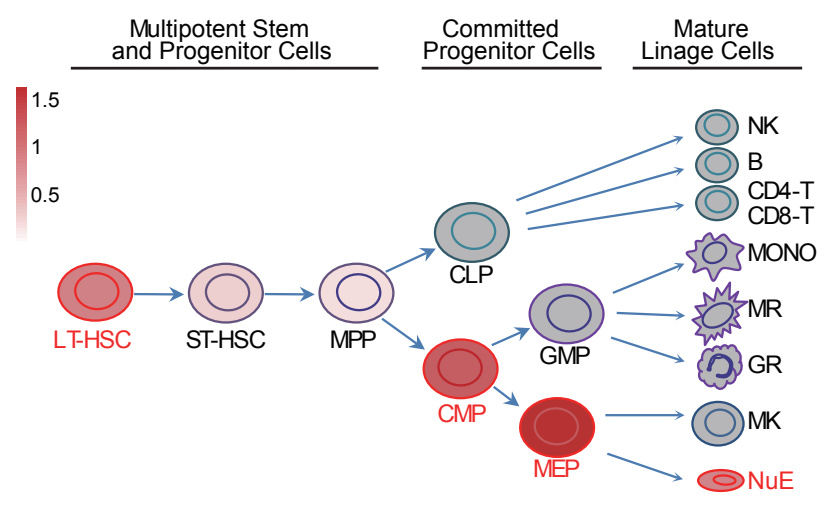

E

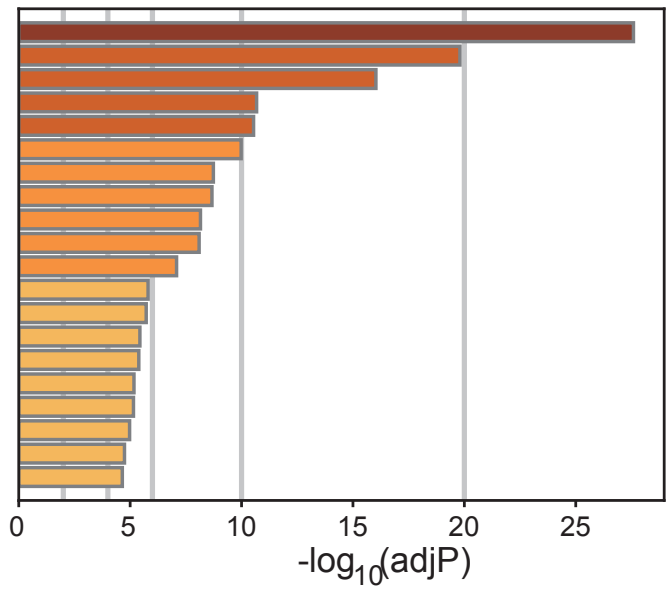

B

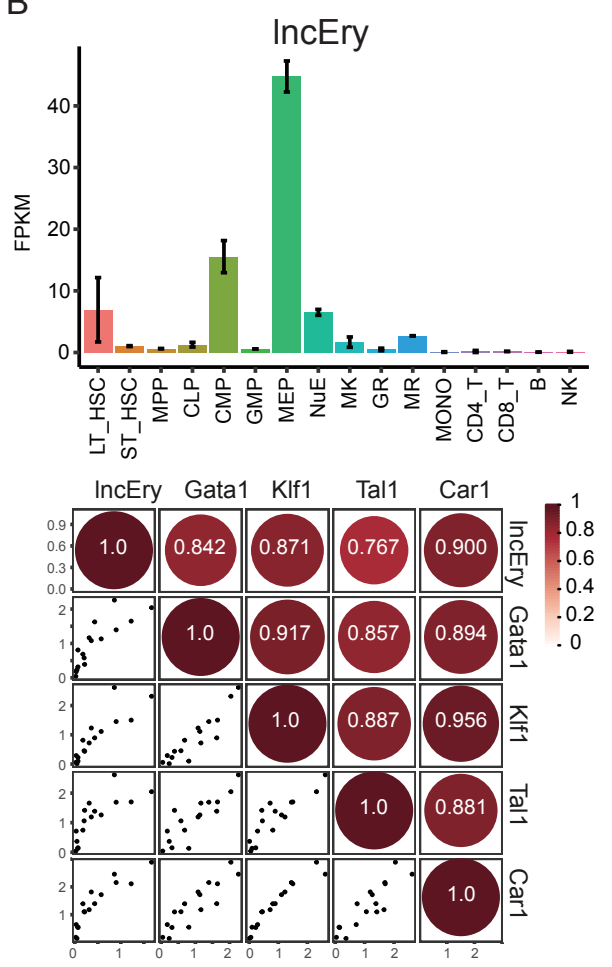

D

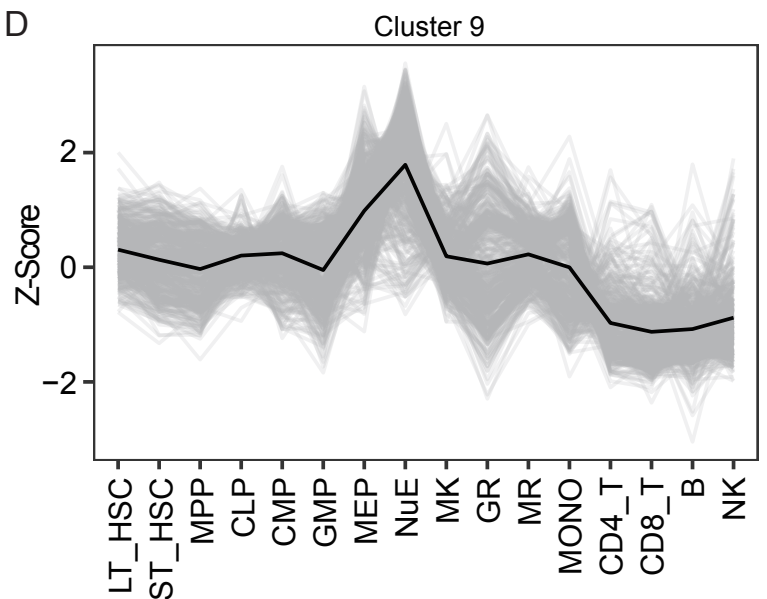

GO:1903047: mitotic cell cycle process GO:0006260: DNA replication GO:0071103: DNA conformation change

GO:0000226: microtubule cytoskeleton organization GO:0000910: cytokinesis

GO:0034101: erythrocyte homeostasis

GO:0030261: chromosome condensation

GO:0050000: chromosome localization

GO:0140013: meiotic nuclear division

GO:0006778: porphyrin-containing compound metabolic process

GO:0061640: cytoskeleton-dependent cytokinesis

GO:0010035: response to inorganic substance

GO:0044839: cell cycle G2/M phase transition

GO:0051383: kinetochore organization

GO:0007098: centrosome cycle

GO:0055076: transition metal ion homeostasis

GO:0090407: organophosphate biosynthetic process

GO:0019755: one-carbon compound transport

GO:0071897: DNA biosynthetic process

GO:0007062: sister chromatid cohesion 
A

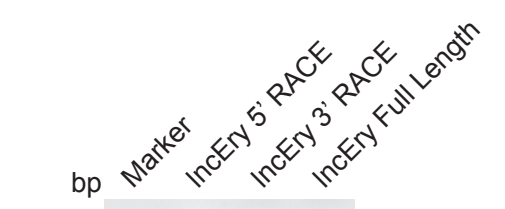

2000 -

1000 -

750 -

500 -

250 -

100 -

C

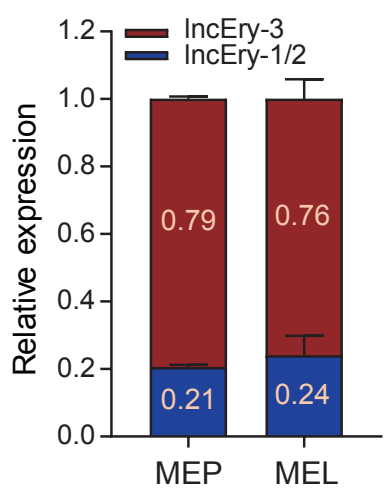

E

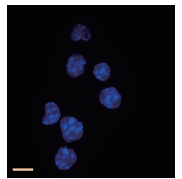

DAPI

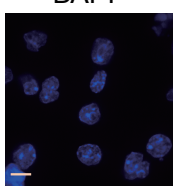

DAPI

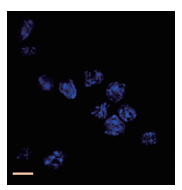

DAPI

D

B
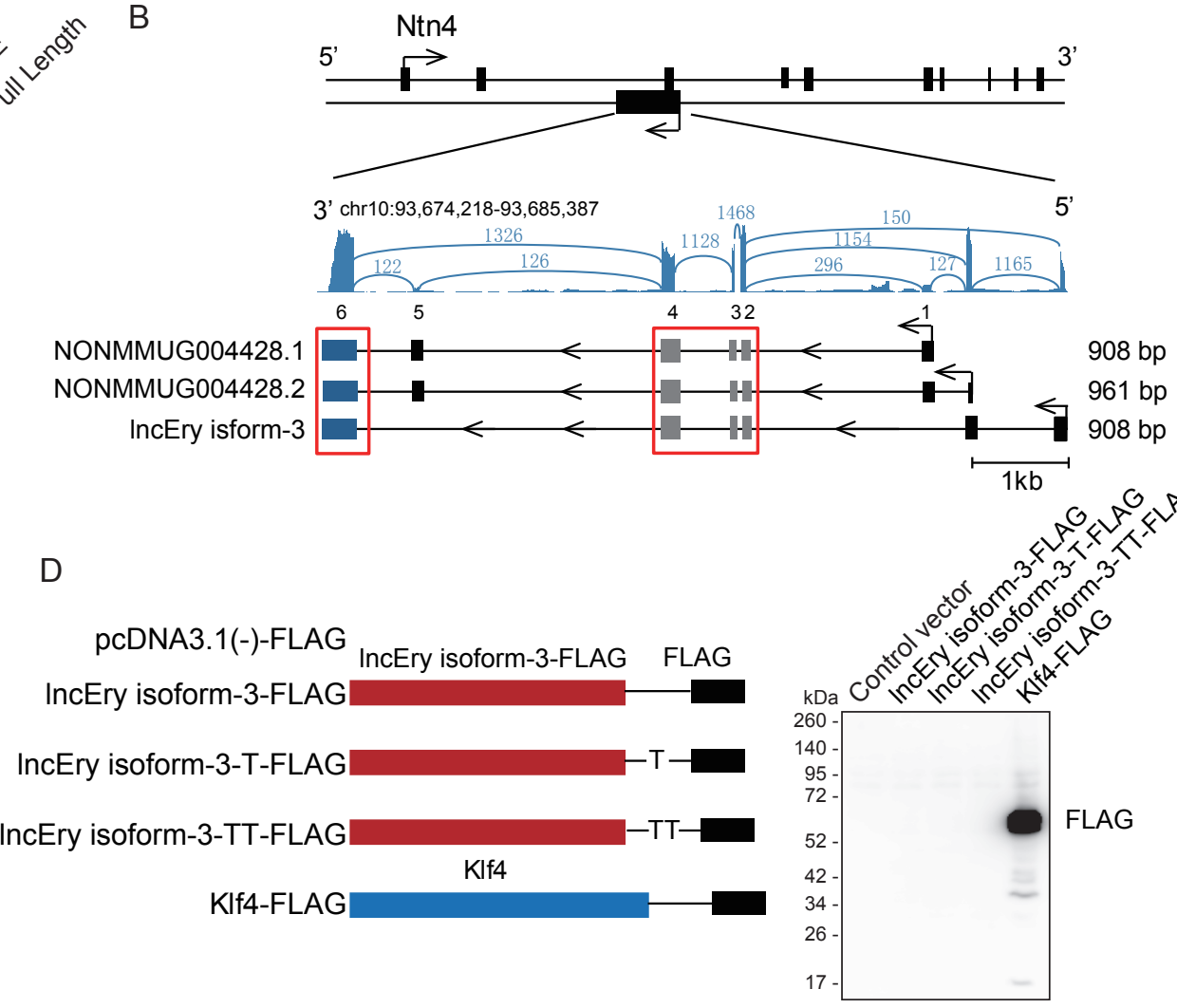

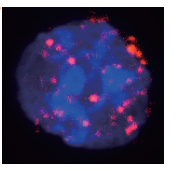

IncEry

Cytosol

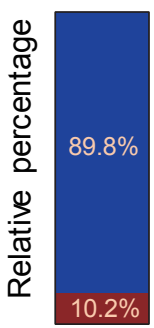

F

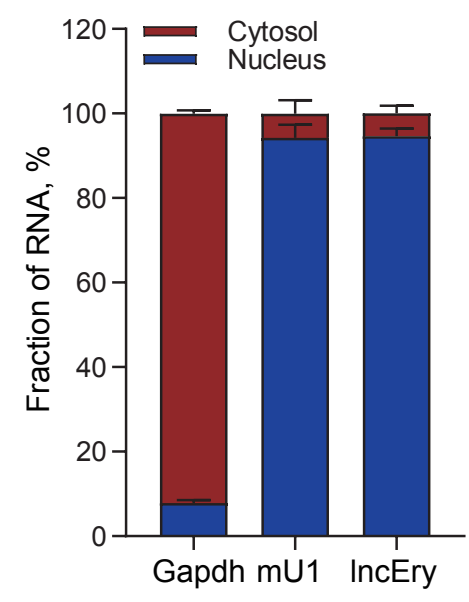

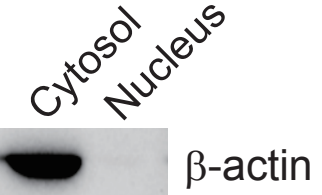

H3 


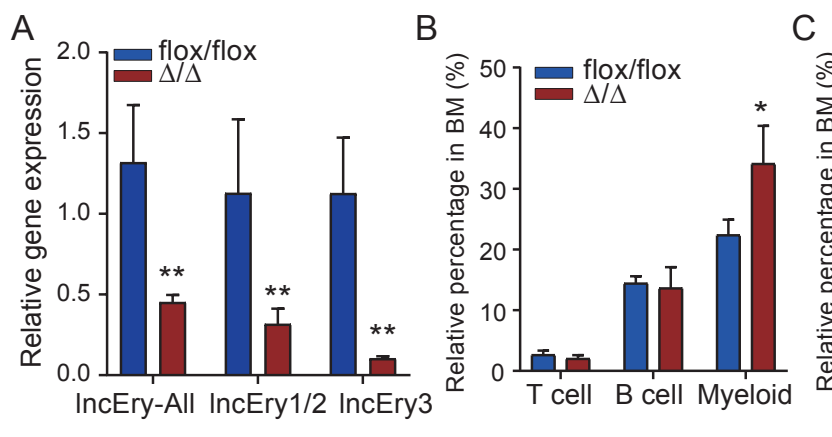

C

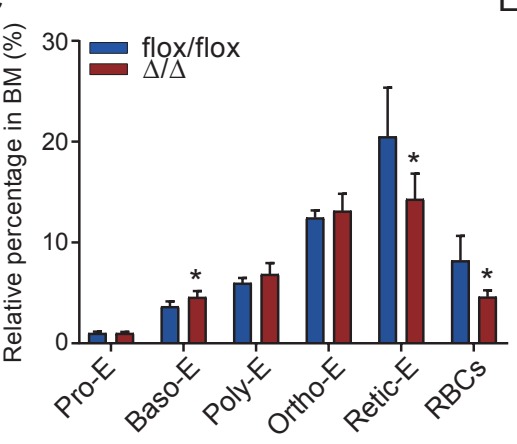

E

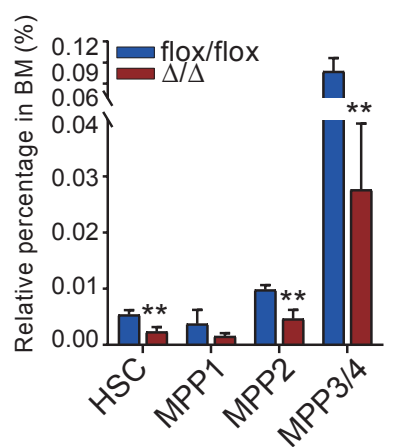

D

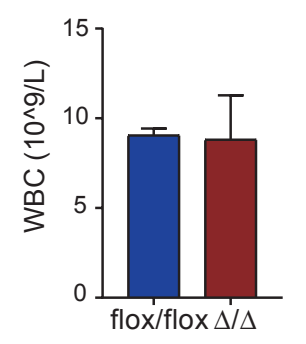

G

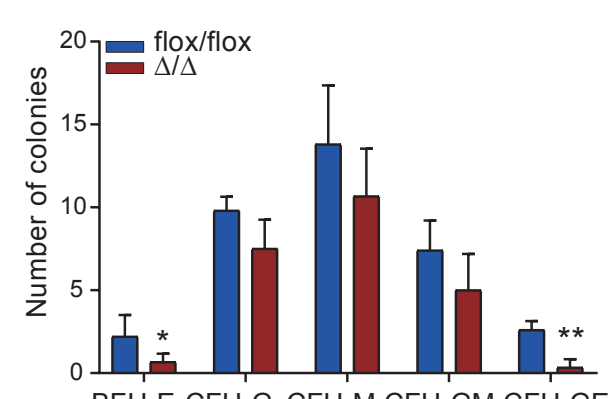

J

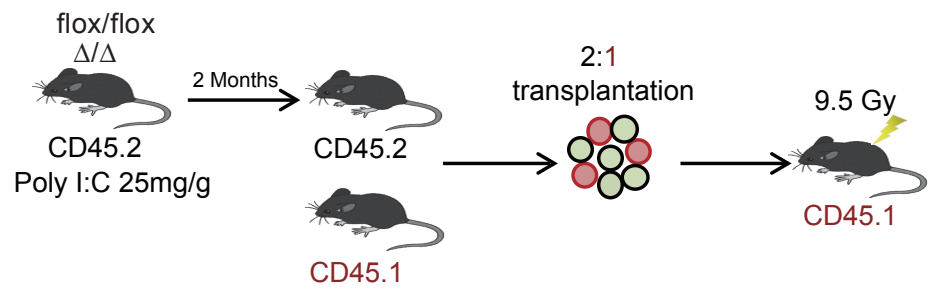

L

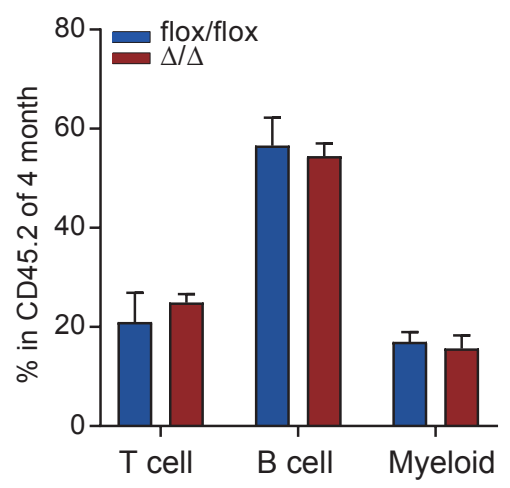

M

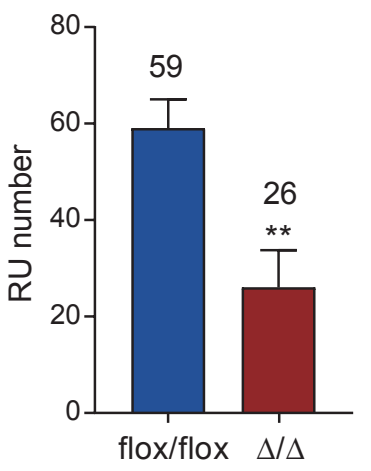

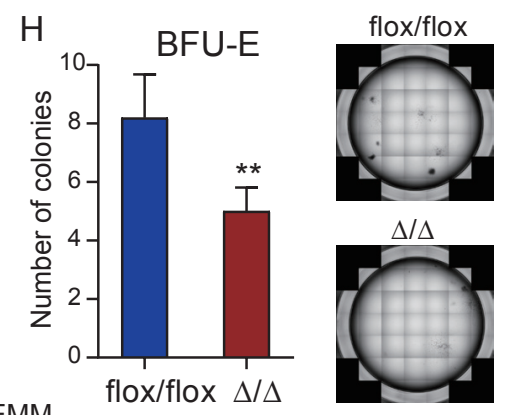
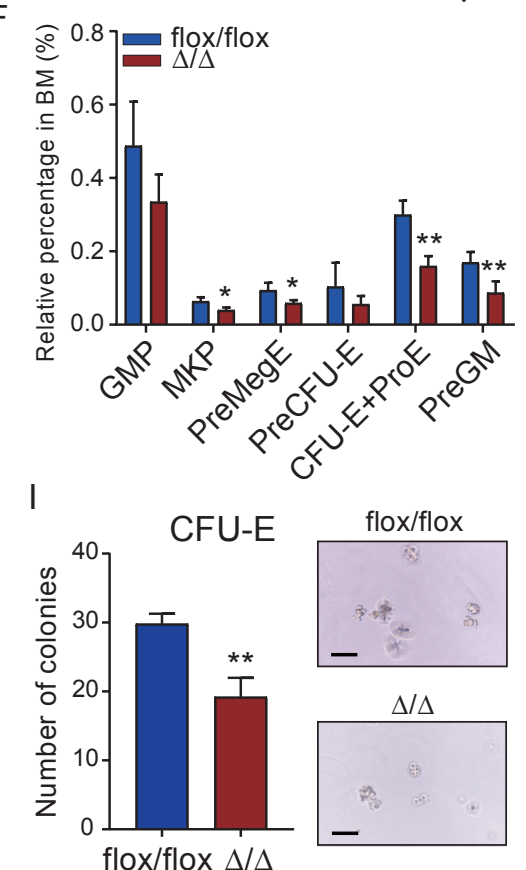

K

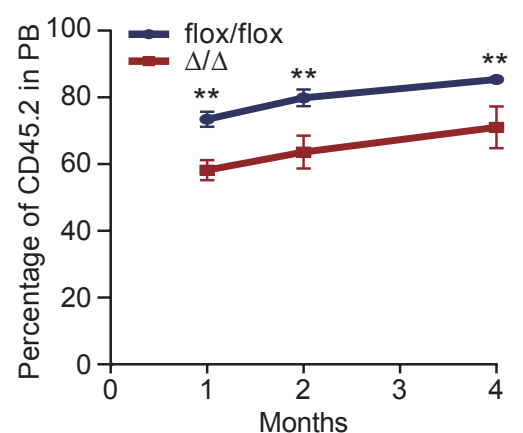


A

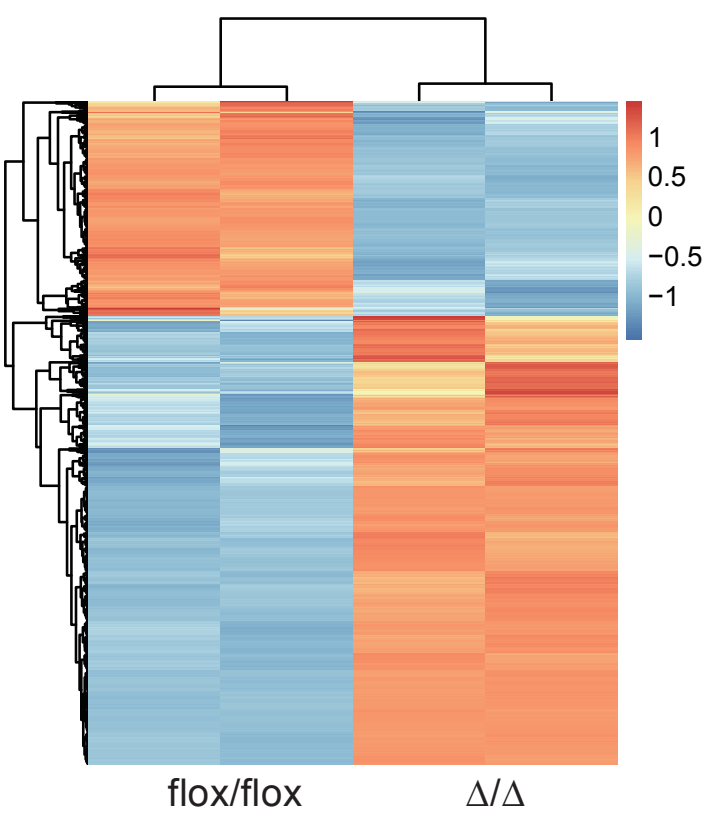

C

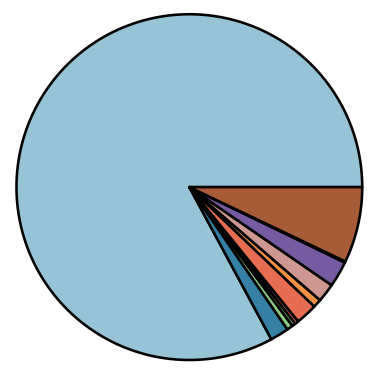

ㅁ Promoter (<=1kb) $(82.87 \%)$

ㅁ Promoter (1-2kb) $(1.74 \%)$

口 Promoter (2-3kb) (0.56\%)

ㅁ 5' UTR $(0.34 \%)$

ㄱ 3' UTR $(0.34 \%)$

ㅁ 1 st Exon $(2.02 \%)$

ㅁ Other Exon $(0.79 \%)$

․ 1 st Intron $(1.74 \%)$

口 Other Intron $(2.42 \%)$

$\square$ Downstream $(<=300)(0.11 \%)$

口 Distal Intergenic $(7.08 \%)$

E

GO Biological Processes

heme metabolic process inorganic ion homeostasis transition metal ion homeostasis erythrocyte differentiation

anatomical structure homeostasis endomembrane system organization regulation of anatomical structure size regulation of anion transport neuron cellular homeostasis mitochondrial transport

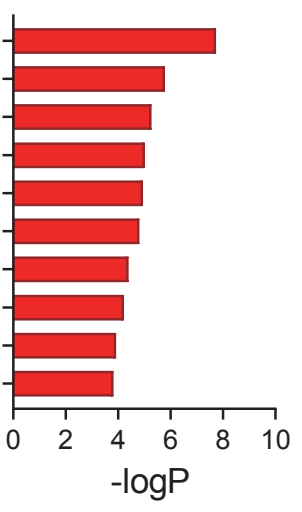

G

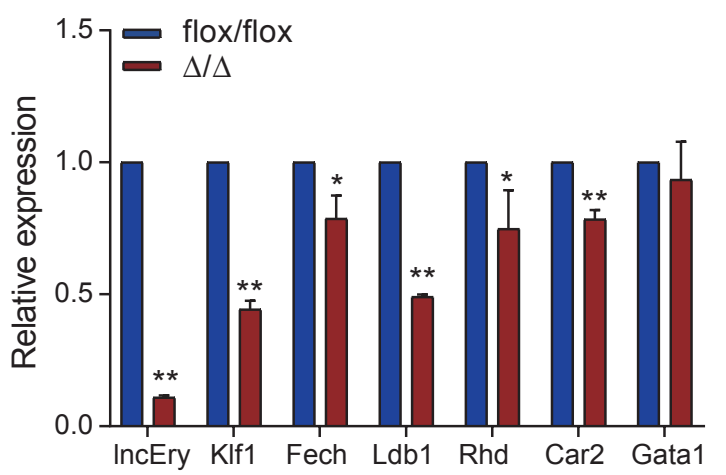

B

F

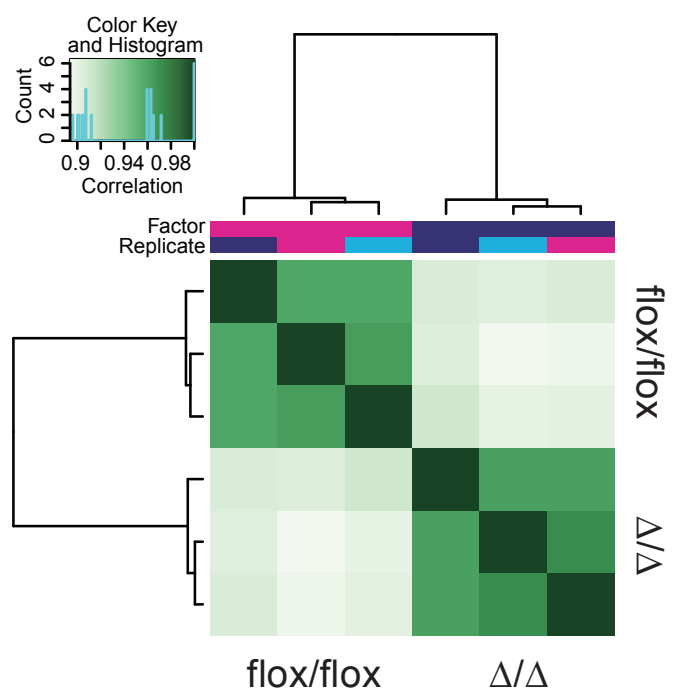

D
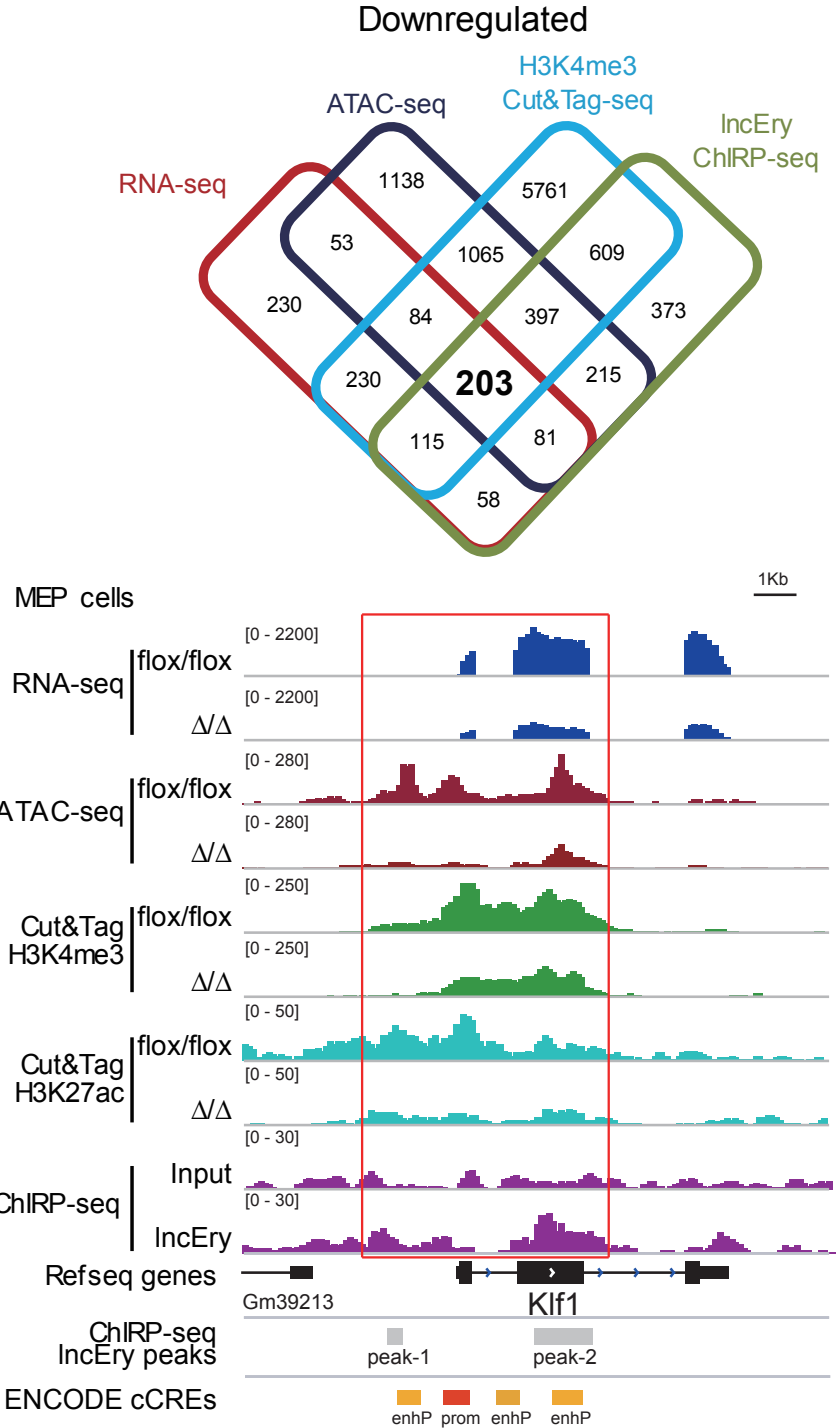
A

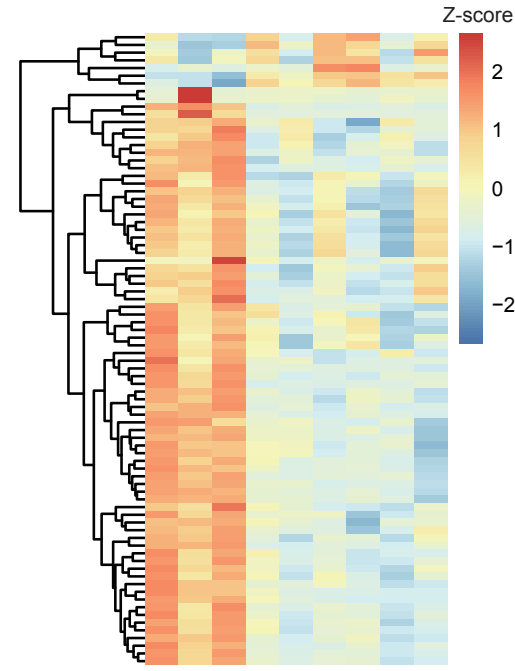

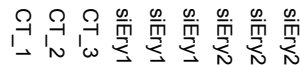

B

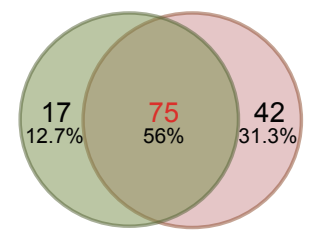

E

siEry-1 vs CT siEry-2 vs CT

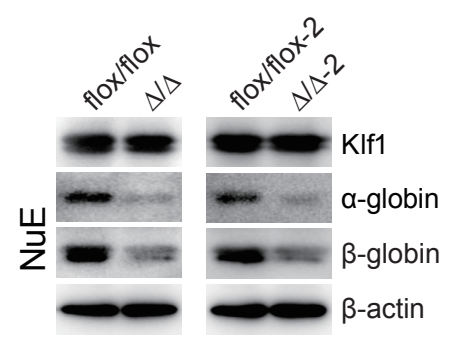

F

Down regulate genes

RNA-seq H3K4me3 ChIP

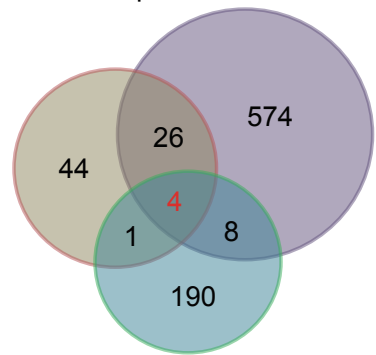

Pol II S5p ChIP
C

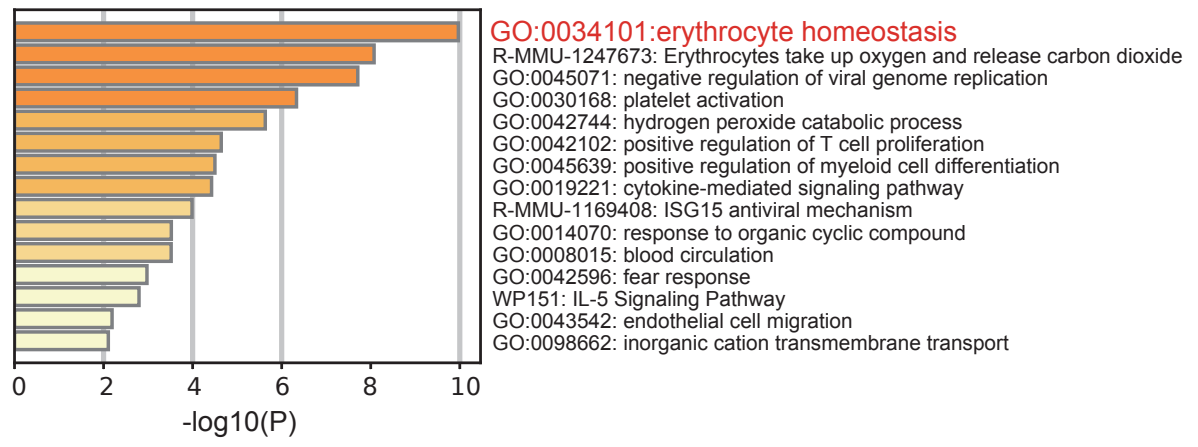

D

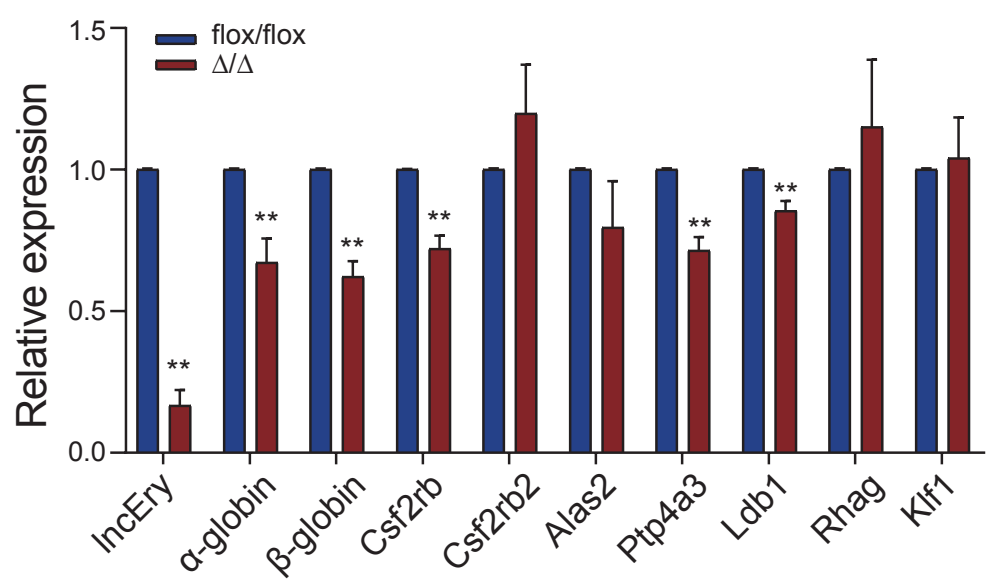

G

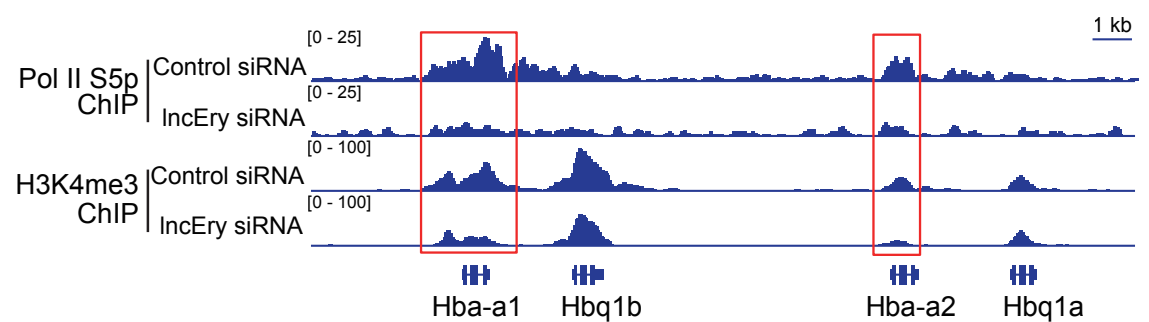

$\mathrm{H}$

Pol II S5p ChIP

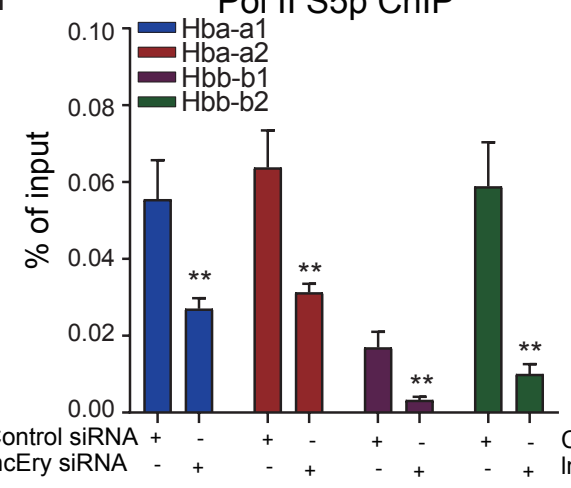

H3K4me3 ChIP

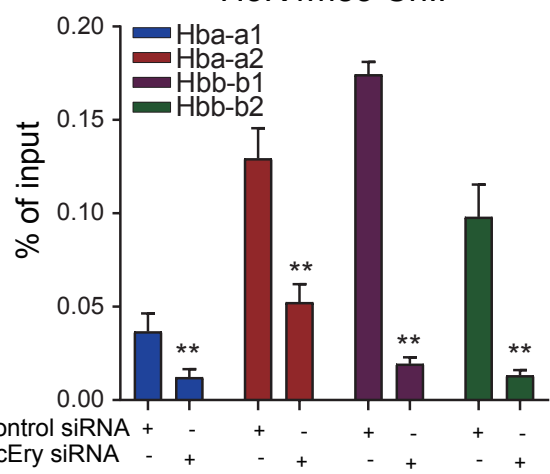


A

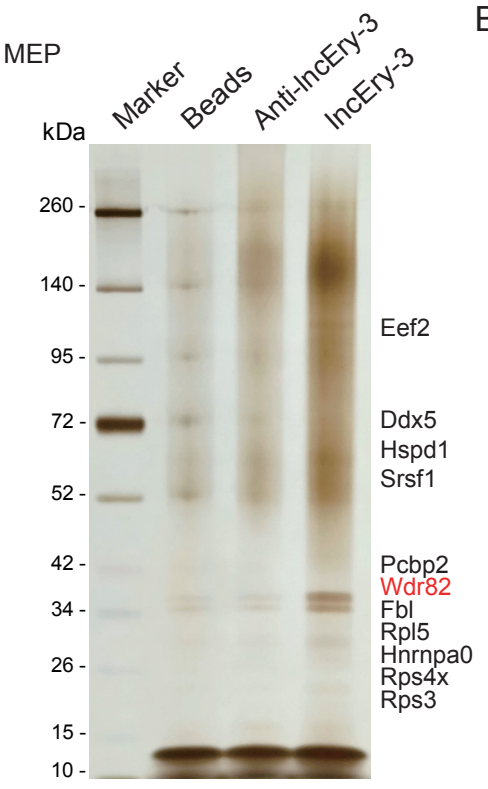

B

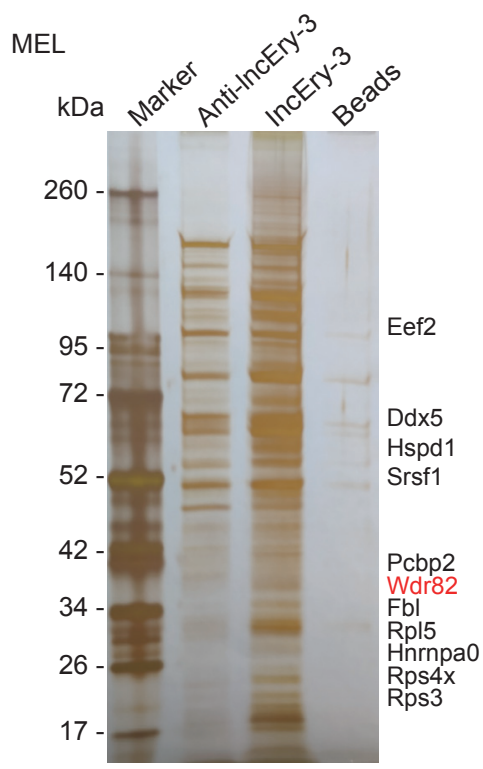

C

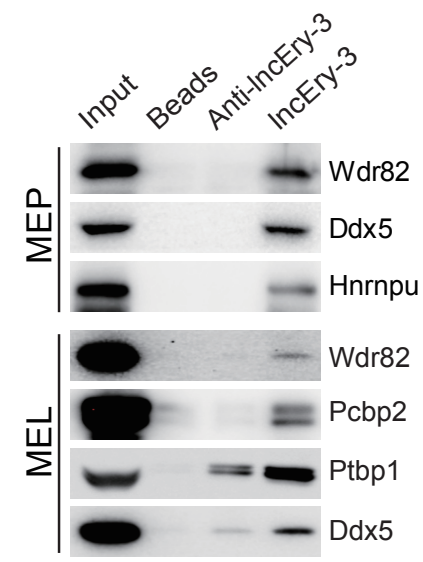

D

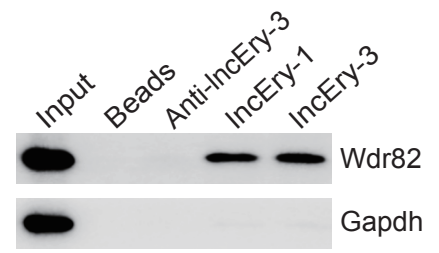

E

$\mathrm{F}$
G
IncEry-P4 IncEry-P5

IncEry-3-Full IncEry-3-P1 IncEry-3-P2 IncEry-3-P3
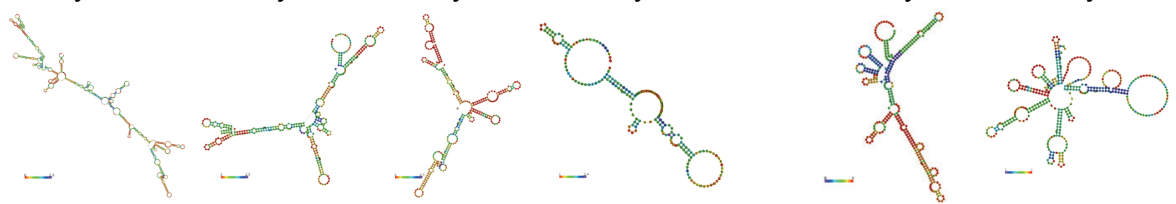

qPCR for Agarose gel electrophoresis
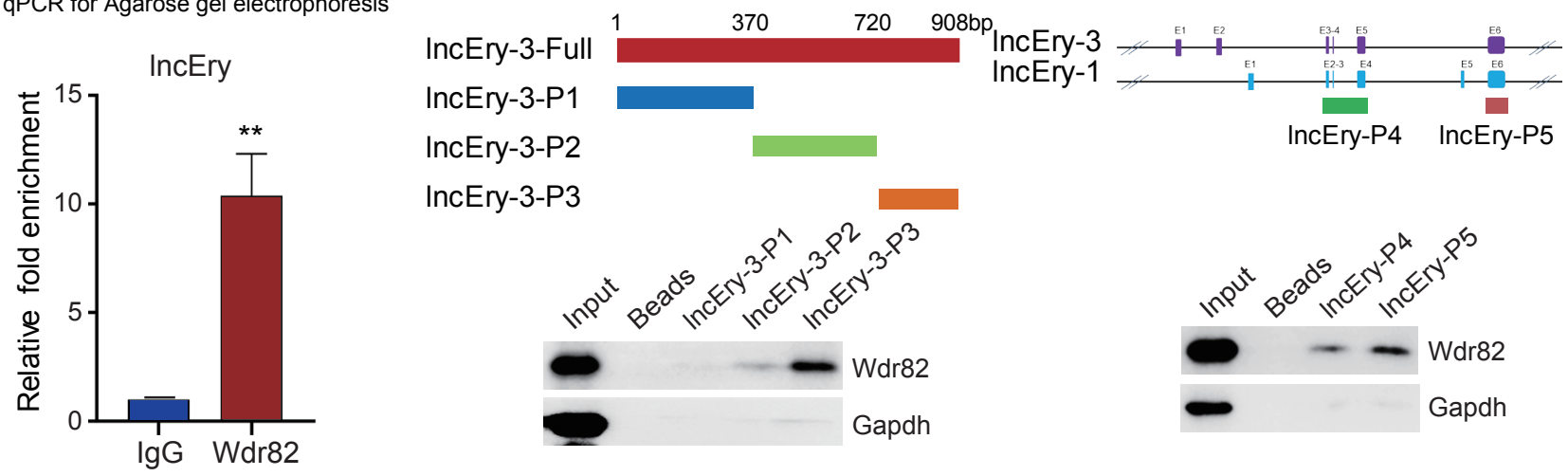

IncEry-3-P3
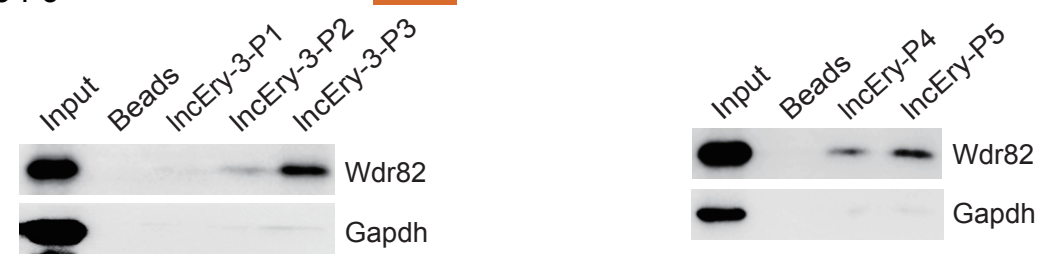

$\mathrm{H}$

IncEry-P5 combined with Wdr82

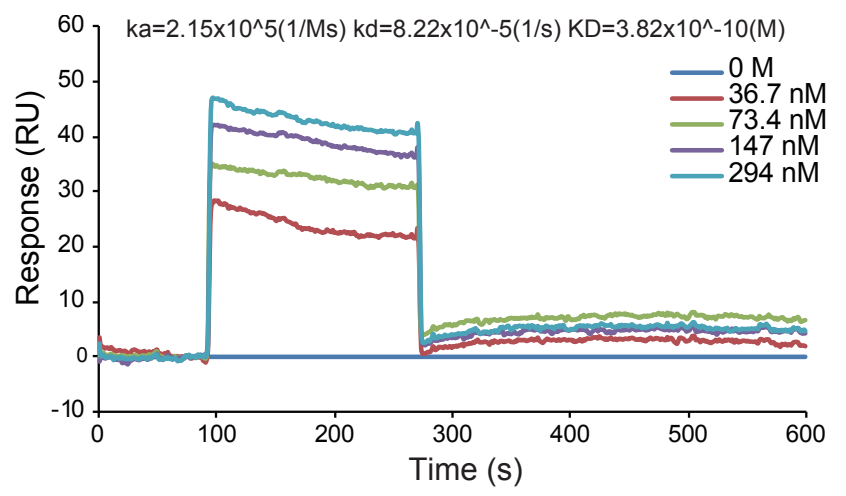

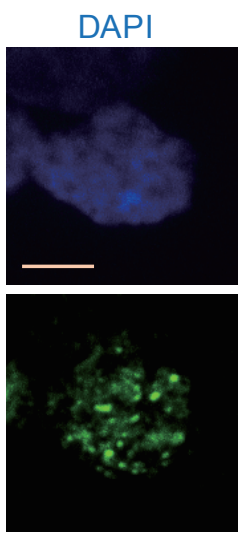

Wdr82
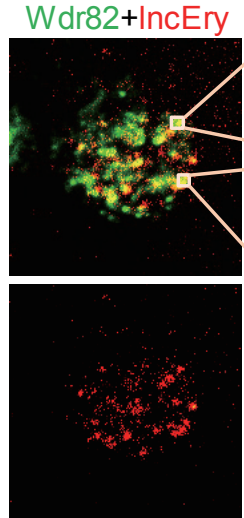

IncEry

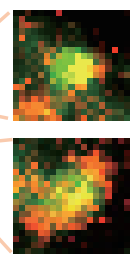

MEP 
A

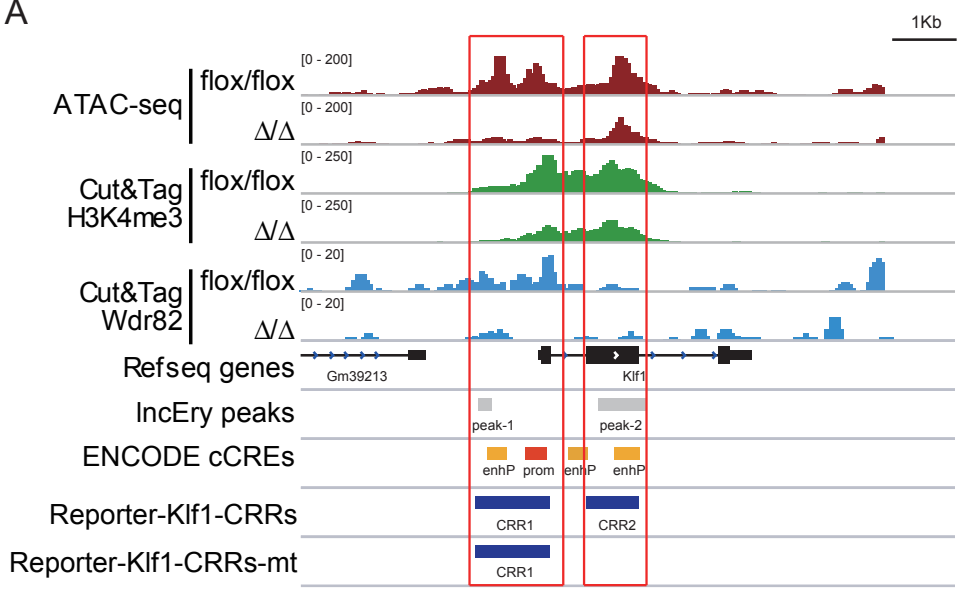

B

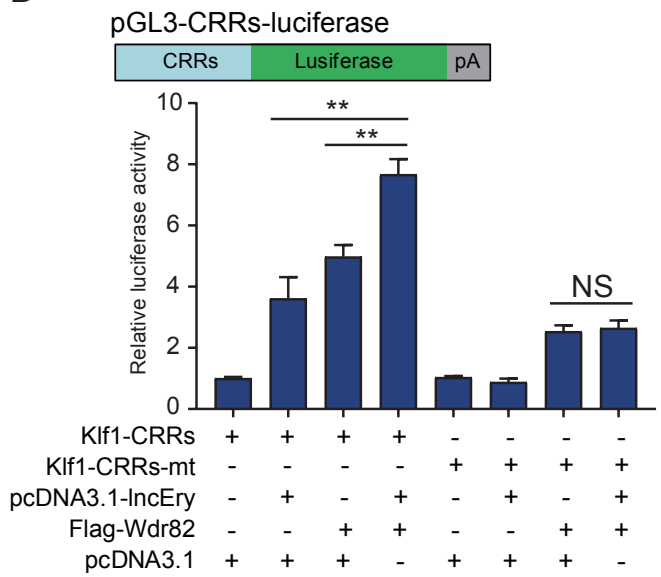

C

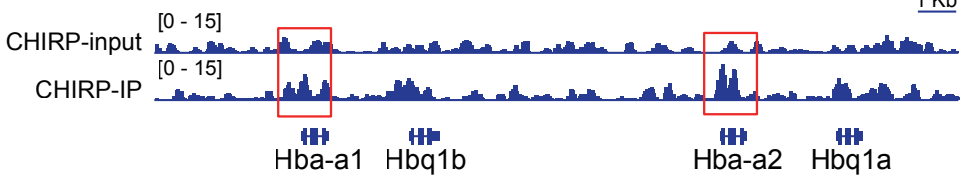

E

pGL3-CRRs-luciferase

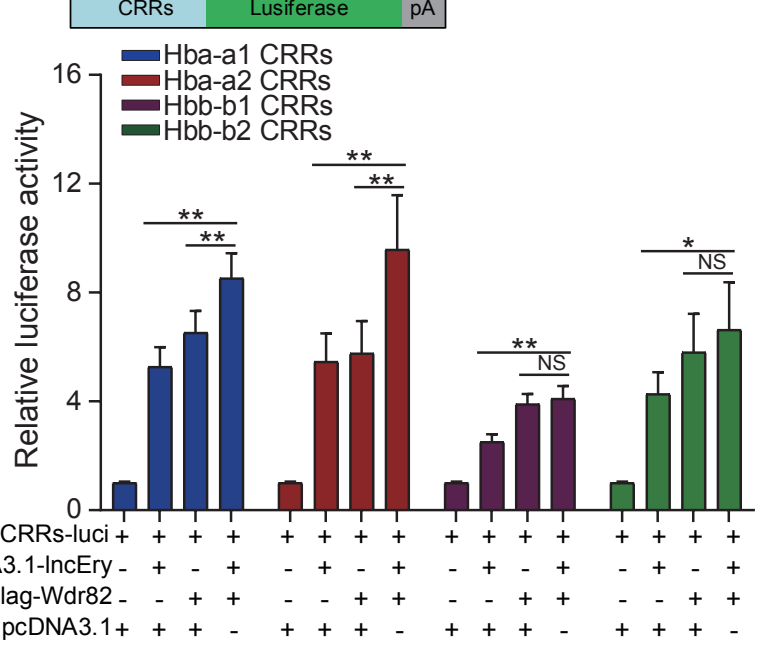

G

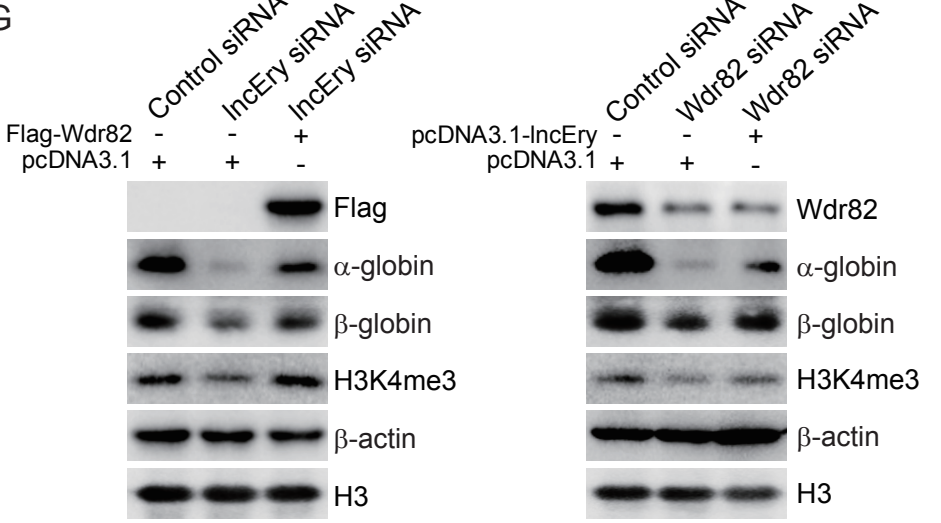

$\underline{1 \mathrm{~Kb}}$

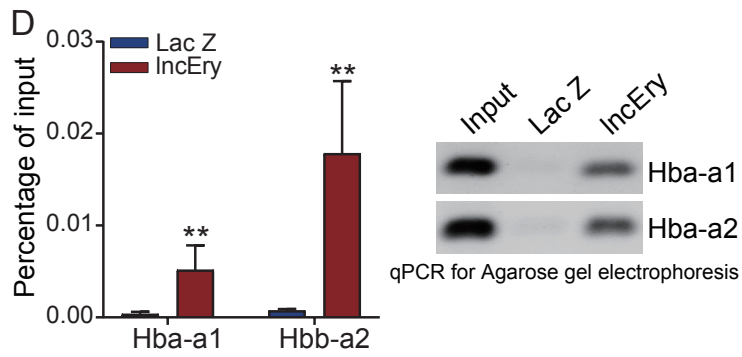

F

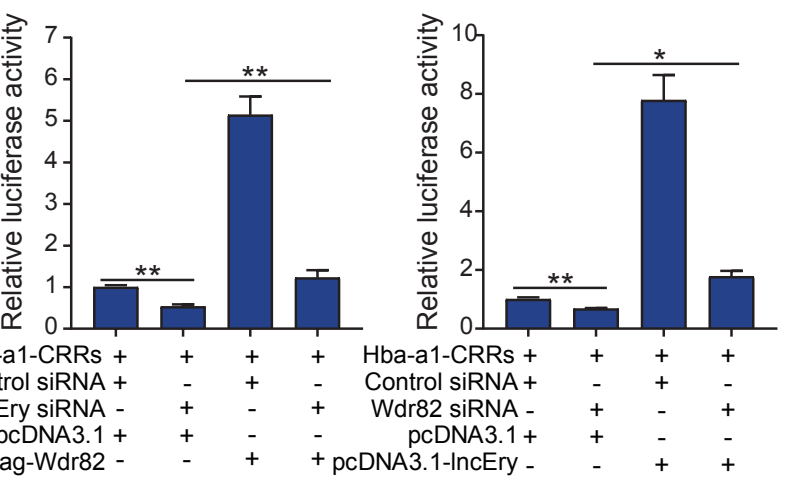

$\mathrm{H}$

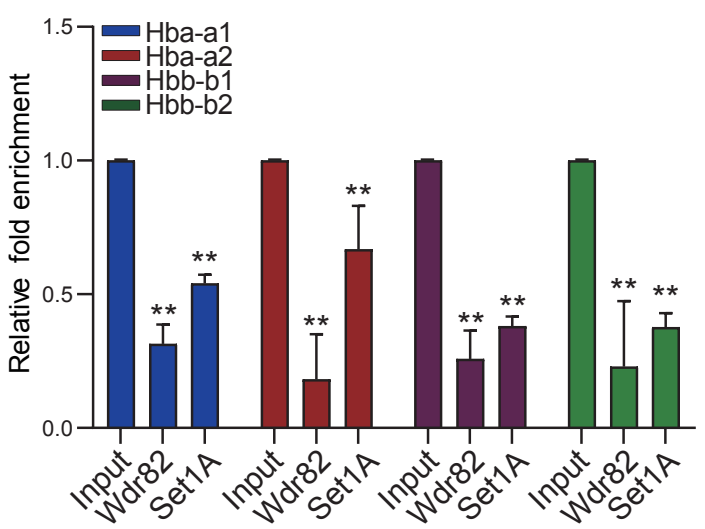


Figure 8

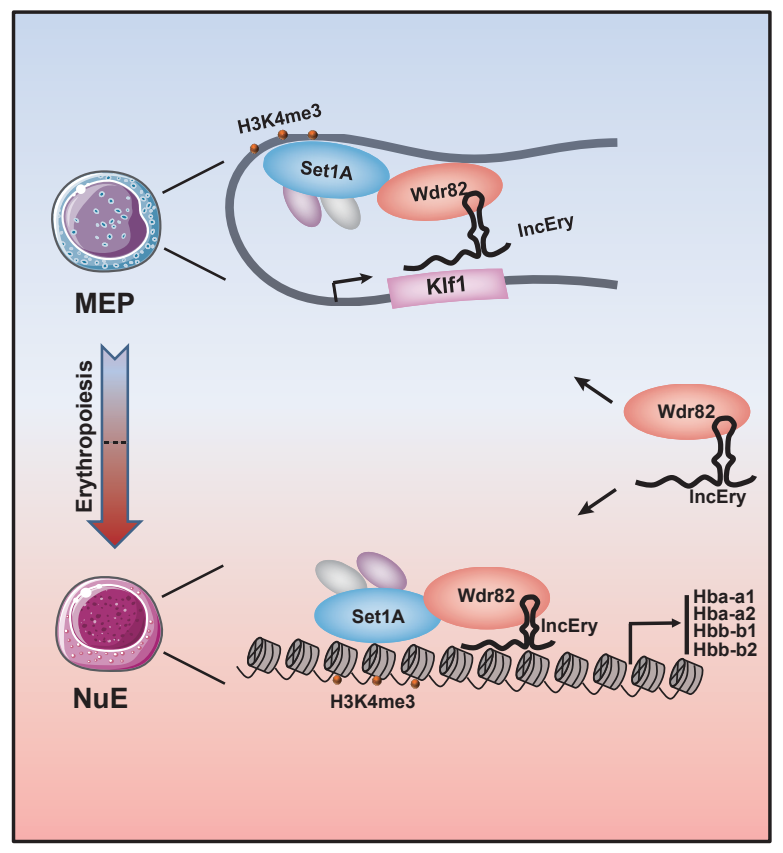

